This item was submitted to Loughborough's Research Repository by the author.

Items in Figshare are protected by copyright, with all rights reserved, unless otherwise indicated.

\title{
A multi-method study of the transformation of the carbonaceous skeleton of a polymer-based nanoporous carbon along the activation pathway
}

PLEASE CITE THE PUBLISHED VERSION

http://dx.doi.org/10.1016/j.carbon.2014.12.051

\section{PUBLISHER}

(C) Elsevier Ltd

VERSION

AM (Accepted Manuscript)

\section{PUBLISHER STATEMENT}

This work is made available according to the conditions of the Creative Commons Attribution-NonCommercialNoDerivatives 4.0 International (CC BY-NC-ND 4.0) licence. Full details of this licence are available at: https://creativecommons.org/licenses/by-nc-nd/4.0/

\section{LICENCE}

CC BY-NC-ND 4.0

\section{REPOSITORY RECORD}

Hu, Cheng, Amelia C.Y. Liu, Matthew Weyland, S. Hadi Madani, Phillip Pendleton, Francisco RodriguezReinoso, Katsumi Kaneko, Mark J. Biggs, and L.A. Smillie. 2019. "A Multi-method Study of the Transformation of the Carbonaceous Skeleton of a Polymer-based Nanoporous Carbon Along the Activation Pathway". figshare. https://hdl.handle.net/2134/18659. 


\title{
A Multi-method Study of the Transformation of the Carbonaceous Skeleton of a Polymer-based Nanoporous Carbon Along the Activation Pathway
}

Cheng $\mathrm{Hu}^{\mathrm{a}}$, Amelia CY Liu ${ }^{\mathrm{b}, \mathrm{c}}$, Matthew Weyland ${ }^{\mathrm{c}, \mathrm{d}}$, S. Hadi Madani ${ }^{\mathrm{e}}$, Phillip Pendleton ${ }^{\mathrm{e}, \mathrm{f}}$, Francisco Rodríguez-Reinoso ${ }^{\mathrm{g}}$, Katsumi Kaneko ${ }^{\mathrm{h}}$ and Mark J. Biggs ${ }^{\mathrm{a}, \mathrm{i}^{*}}$

a School of Chemical Engineering, The University of Adelaide, SA 5005, Australia.

b School of Physics, Monash University, Victoria, 3800, Australia.

${ }^{c}$ Monash Centre for Electron Microscopy, Monash University, Victoria, 3800, Australia.

${ }^{\mathrm{d}}$ Department of Materials Engineering, Monash University, Victoria, 3800, Australia.

e Ian Wark Research Institute, University of South Australia, SA 5095, Australia.

f Sansom Institute, University of South Australia, SA 5001, Australia.

g. Laboratorio de Materiales Avanzados, Departamento de Química Inorgánica, Universidad de Alicante, Apartado 99 E-03080, Spain.

h Center for Energy and Environmental Science, Shinshu University, Wakasato 4-17-1, Nagano 380-8553, Japan.

${ }^{\text {i }}$ School of Science, Loughborough University, Leicestershire, UK, LE11 3TU.

\begin{abstract}
The change in the carbonaceous skeleton of nanoporous carbons during their activation has received limited attention, unlike its counterpart process in the presence of an inert atmosphere. Here we adopt a multi-method approach to elucidate this change in a poly(furfuryl alcohol)-derived carbon activated using cyclic application of oxygen saturation at $250^{\circ} \mathrm{C}$ before its removal (with carbon) at $800^{\circ} \mathrm{C}$ in argon. The methods used include helium pycnometry, synchrotron-based X-ray diffraction (XRD) and associated radial distribution function (RDF) analysis, transmission electron microscopy (TEM) and, uniquely, electron energy-loss spectroscopy spectrum-imaging (EELS-SI), electron nanodiffraction and fluctuation electron microscopy (FEM). Helium pycnometry indicates the solid skeleton of the carbon densifies during activation from $78 \%$ to $93 \%$ of graphite. RDF analysis, EELS-SI, and FEM all suggest this densification comes through an in-plane growth of $s p^{2}$ carbon out to the medium range without commensurate increase in order normal to the plane. This process could be termed 'graphenization'. The exact way in which this process occurs is not clear, but TEM images of the carbon before and after activation suggest it may come through removal of the more reactive carbon, breaking constraining cross-links and creating space that allows the remaining carbon material to migrate in an annealing-like process.
\end{abstract}

*m.biggs@lboro.ac.uk 


\section{Introduction}

The study of the solid skeleton of carbonaceous materials is as long standing as X-ray diffraction (XRD) [1]. Whilst the study of the evolution of the carbonaceous skeleton during processing has a somewhat shorter history [1], it is still well developed for the case where the carbons and their precursors are heated in an inert atmosphere (i.e. in pyrolysis/carbonization and graphitization) (e.g. [2-17]). Far less effort has, however, been directed towards elucidating the evolution of the skeleton when heating occurs in the presence of oxidizing gases (i.e. during gasification/activation processes), in stark contrast to the accompanying porosity change. There is now a growing realization that there are two sides to the gasification/activation story [18-22]: one must consider the evolution of the carbonaceous skeleton as well as the porosity that it defines in order to have a complete picture.

The earliest work concerned with elucidating the change in the solid skeleton of carbonaceous materials when heated under an oxidative environment appears to be that of Noda and Inagaki [23], who compared XRD-derived quantities for carbons obtained from heating under inert and oxidative environments. Based on the graphitic crystallite model of Biscoe and Warren [2], for the same heat treatment temperature, they found oxidative environments yielded crystallites with a greater number of layers and a smaller inter-layer spacing. They hypothesized this oxidative enhancement of 'graphitization' came from the oxygen removing more reactive cross-links between the graphitic crystallites in preference to the less reactive crystallites themselves, allowing them to then go on and grow, presumably through some rearrangement and fusion process akin to annealing. Behavior similar to that seen by Noda and Inagaki was observed 25 years later by Levendis and Flagan [24] for a glassy carbon derived from furfuryl alcohol (FA), the material of particular focus in the study reported here. Although they offer up nothing new on the possible origins of this behavior, they do proffer support for it in the form of transmission electron microscopy (TEM) images of the carbon heat treated in nitrogen and air.

The next body of work in the field appears to have centered on understanding gasificationinduced densification, which was first posited when char particles were observed to shrink whilst being gasified under kinetically controlled conditions. This phenomenon was first alluded to by Johnson [18] (see also pg. 1524 of [25]), who considered gasification of various $400-800 \mu \mathrm{m}$ sized coal chars in hydrogen and steam at 35 bar and 1200 K. Hurt and co-workers [19] undertook a more comprehensive study a decade later by studying the gasification of a variety of fine (less than $38 \mu \mathrm{m}$ ) chars, including Spherocarb, under kinetic control. Johnson attributed densification during gasification to 'reorientation of individual carbon crystallites' (there is also mention of growth in the crystallite dimensions in the context of surface area decrease with conversion in one coal char). Hurt and co-workers went further, however, by hypothesizing atomic-scale rearrangement of the solid atoms following removal 
by gasification of some atoms and cross-links between sections of the skeleton. Indirect support for this hypothesis was provided subsequently by the work of Kantorovich and Bar-Ziv in which a model that appears to capture the essentials of the hypothesis was able to reproduce experimentally observed changes in porosity [21], reactivity [26] and thermal conductivity [27] with char conversion during gasification.

Further understanding of the change in the carbonaceous skeleton under oxidative conditions has come from some of the work focused on elucidating the underlying causes of char reactivity variation during coal gasification and combustion [28-36]. This work clearly shows that the nature of the change in extent of order in the skeleton with conversion is dependent on the char and the conditions it experiences: most of the studies demonstrated an increase in extent of order with conversion [28-36], but others also saw a decrease [28, 35] or no change at all [34, 35]. The more recent work of Feng et al. [22], however, raises the prospect that conclusions drawn from this prior work may be open to question if based solely on XRD analysis. As an indirect method, XRD is also not generally well placed to elucidate the underlying mechanism of the change in the carbonaceous skeleton. The use of multiple techniques together and/or more direct methods such as TEM is likely to be more fruitful.

A small number of the studies that have adopted a multi-technique or direct analysis approach to studying change in the carbonaceous skeleton with conversion have proposed mechanisms for the change. Based on the Oberlin basic structural unit (BSU) model [37], which is akin to the graphitic microcrystallite model [2], and their assembly into regions of local molecular orientation (LMO) [6], Rouzaud et al. [20] used quantitative TEM of petroleum coke at various levels of conversion from steam gasification at $950^{\circ} \mathrm{C}$ to propose that, except at high conversions, regions of LMO of intermediate size (10-100 nm) were preferentially consumed over the larger and smaller regions of LMO. They attributed this behavior to a balance between the accessibility of the oxidant to the solid atoms, which increases with the extent of LMO, and their reactivity, which decreases with the LMO extent. However, more recent studies involving coal chars [36] and coke [38] argue that the smallest graphitic crystallites are preferentially consumed, leaving behind the larger crystallites, which can be equated to all but the smallest of Oberlin's regions of LMO. Beyond this earlier work, some studies [33, 36, 38] support the earlier hypothesis $[19,21,23]$ that disordered carbon is preferentially consumed. They, on the other hand, offer mixed support for the previously hypothesized notion $[18,19,21,23]$ that removal of the more reactive carbon allows the remainder to go on and anneal - Tran et al. [38] ruled this possibility out, claiming that the more ordered carbon already existed in their precursor (coke), whilst Sharma et al.[33] argued that the ordered structures did not initially exist in their coal char but formed through an oxidative-assisted annealing process. 
To a significant extent, the current modest understanding of the fundamentals of carbon skeleton evolution under oxidative environments arises out of the limitations of the various experimental techniques and approaches used to date. In the case of the most common XRD-based analysis, the most fundamental concern is its reliance on the view that carbons are composed of nanoscale graphitic crystallites, something that has been questioned many times [39], most recently with the support of unprecedented TEM images that demonstrate the skeleton of activated carbons contain 5- and 7-membered rings and associated curvature [40, 41]. Even if the crystallite model were to be accepted, many of these XRD analyses assume line broadening in the diffraction patterns is due to just one or two origins, omitting many others [39]. The exception to this is the approach of Shi et al. [42], but this is far more difficult to apply and does not appear to be always reliable [22]. An alternative to these graphitic crystallite-based approaches is radial distribution function (RDF) analysis [1]. This approach has the advantage that it is not dependent on a specific model of the carbon skeleton. The quality of RDF analysis is, however, strongly constrained by the quality of the scattering data and its treatment [43]. Its capacity to elucidate the atomic structure of noncrystalline materials like those of interest here is also limited to short length scales; just a few bond lengths.

The more direct nature of TEM means it does not carry the various concerns that accompany XRD. However, quantification of TEM images, which is essential to more objective analysis of carbon structure and its evolution, is also not without problems. For example, no matter how good the microscope, the projection of even very thin (e.g. $2 \mathrm{~nm}$ ) volumes of disordered materials on to planes as done in TEM can yield fringes that are suggestive of order that does not in fact exist [44]. Beyond these imaging issues, if quantification is attempted, it requires various image processing operations that are open to uncertainty. Even if these operations were perfect, however, it does not provide a capacity to determine an absolute fraction of, for example, ordered and disordered material as the nature of the areas of a micrograph that contain no fringes is unknown - they could be solid that is simply not visible under the imaging conditions or true voids [34]. TEM images suitable for quantitative analysis also, of course, run the risk of being less than representative of the bulk material because they are only a few 10 s of $\mathrm{nm}$ in size and limited to very thin regions of material [44].

Scanning transmission electron microscopes (S/TEM) facilitate a multi-method approach by allowing simultaneous TEM, fluctuation electron microscopy (FEM) and electron energy-loss spectroscopy spectrum-imaging (EELS-SI) to be undertaken with ease across mesoscopic areas. Whilst the first of these approaches has been widely used to study carbon structure, the other two have to date not been applied to nanoporous carbons as far as we are aware. Despite this, they have much to offer here. FEM [45] uses spatially resolved electron nanodiffraction data from a large nanoscale area $\left(\right.$ e.g. $\left.30 \times 30 \mathrm{~nm}^{2}\right)$ to probe 
the so-called medium-range (5-30 $\AA$ ) order (MRO) of the solid structure, well beyond that which can be explicitly elucidated by XRD or selected area electron diffraction (SAED). It has been successfully used to study MRO in a range of materials [45], including amorphous carbon films [46, 47], and graphitic carbons [48]. EELSSI uses spatially resolved EELS to determine maps of $s p^{2}$ carbon content across nanoscale areas comparable to that used in FEM. This mapping of the carbon hybridization across a large volume enables not only the accurate quantitative determination of the nature of the carbon atoms and its variability, but also its spatial distribution, including the level of spatial homogeneity. This data complements and enriches the data obtained from TEM, XRD, and FEM. Once again, whilst the long-standing EELS method has been applied to a range of carbon materials (e.g. [49-53]), to our knowledge EELS-SI has not been applied to nanoporous carbons such as those considered here.

Here we report the use of TEM, FEM and EELSSI together with synchrotron-based XRD analysis to study the change in the structure of a nanoporous carbon at three points along the activation pathway - at the start, around 45\% conversion, and approximately halfway inbetween (around 25\% conversion). As far as we are aware, this is the first time that such a multitechnique approach has been used to elucidate the change in the skeleton of a nanoporous carbon when exposed to an oxidative environment.

\section{Experimental Details}

\subsection{Carbon material}

In order to reduce as much as possible experimental uncertainties, the carbon studied here was derived from a furfuryl alcohol (FA) precursor using a carefully controlled process. The full details of the synthesis process may be found elsewhere [54] but are briefly outlined here for convenience. The as-received FA was vacuum-distilled to remove any contaminants before being mixed under an inert atmosphere with oxalic acid dehydrate as a polymerization catalyst. Small batches of the mixture were then heated in a horizontal quartz tube-furnace to $150^{\circ} \mathrm{C}$ under continuous argon flow before being soaked for 1 hour to bring about polymerization. Carbonization was then done under argon by further increasing the temperature to $800^{\circ} \mathrm{C}$ before being soaked for 2 hours. The cooled char was then broken up into chunks using a clean zirconia press before being ball-milled and sieved to obtain a powder with a particle size distribution of 38-106 $\mu \mathrm{m}$. The powdered material was then activated using a cyclic $\mathrm{O}_{2}$ activation protocol that involves repeated application of a cycle in which oxygen is first chemisorbed onto the carbon at $250^{\circ} \mathrm{C}$ and then removed along with some of the carbon (mainly as $\mathrm{CO}$ and $\mathrm{CO}_{2}$ ) at $800^{\circ} \mathrm{C}$ in an inert atmosphere. Three materials along the activation pathway were considered: (a) the unactivated char (denoted C0 henceforth), (b) that corresponding to $25 \%$ conversion (denoted $\mathrm{C}_{2} 5 \mathrm{O}_{2}$ henceforth [55]), and (c) that corresponding to $45 \%$ conversion (denoted $\mathrm{C}_{45} \mathrm{O}_{2}$ henceforth [55]). 
Previous work by us [54, 56] has demonstrated that both the porosity and chemistry, a summary of which may be found in Fig. 1 and Table 1, is homogeneous within the particles.
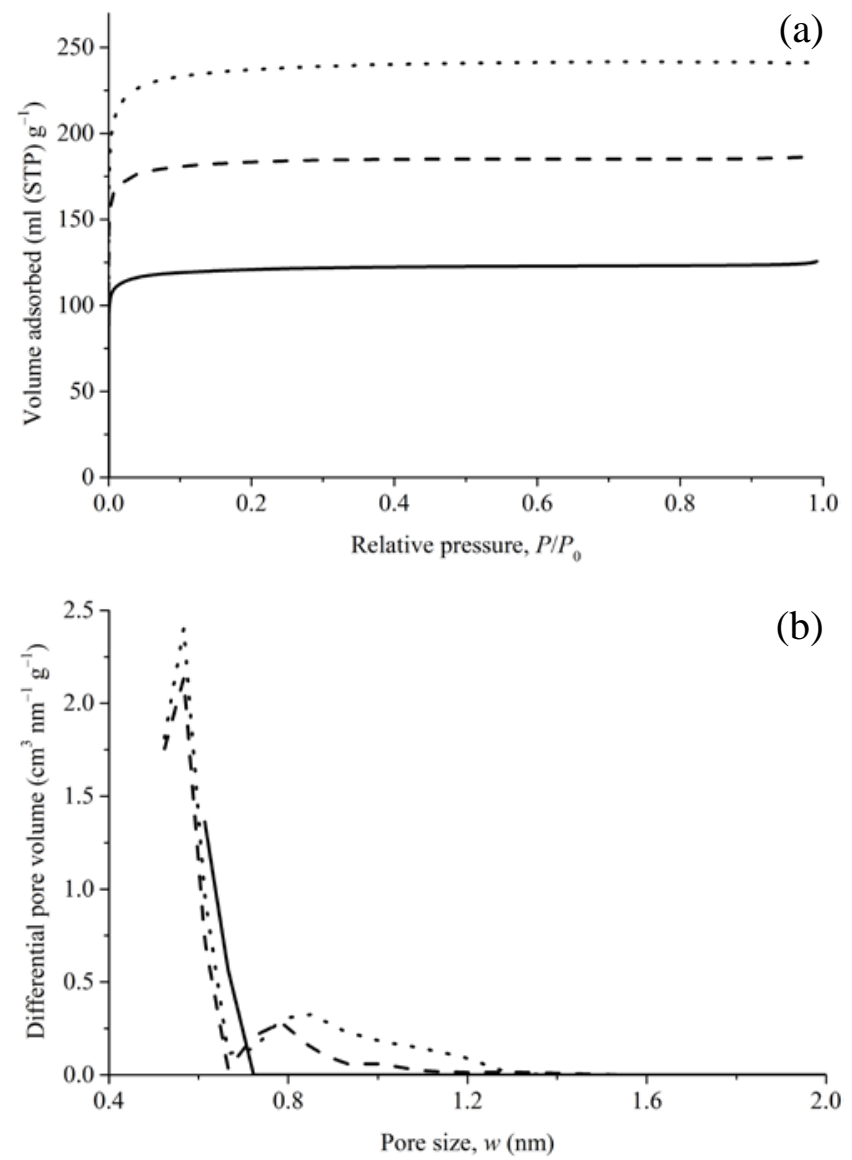

Fig. 1. Porosity characterisation of carbons $\mathrm{CO}$ (solid line), $\mathrm{C}_{25 \mathrm{O}_{2}}$ (broken line) and $\mathrm{C}_{45} \mathrm{O}_{2}$ (dotted line): (a) $\mathrm{N}_{2}$ adsorption isotherms at $77 \mathrm{~K}$; and (b) pore size distributions from the isotherms using QSDFT method [57]. The quality of the PSD for the C0 carbon is modest due to the low number of points determined for the isotherm, which resulted from the very long ( $\sim 1$ week) equilibration time required for each point.

For the S/TEM-based characterisation, the powdered carbon was further mildly ground with spectroscopic grade ethanol in a mortar and pestle and then sonicated. The sample suspensions were allowed to settle and then the top fraction was dropped onto copper TEM grids coated with a holey carbon support. The grids were kept under vacuum prior to insertion into the S/TEM.
Table 1. Porosity and chemistry characteristics of the three carbons studied here.

\begin{tabular}{cccccc}
\hline Carbon & $\begin{array}{c}\mathbf{S S A}^{\mathbf{a}} \\
\mathrm{m}^{2} / \mathrm{g}\end{array}$ & $\begin{array}{c}\mathbf{S P V}^{\mathbf{b}} \\
\mathrm{cm}^{3} / \mathrm{g}\end{array}$ & $\begin{array}{c}\mathbf{C}^{\mathrm{c}, \mathrm{d}} \\
\mathrm{wt}^{-} \%\end{array}$ & $\begin{array}{c}\mathbf{H}^{\mathrm{c}, \mathrm{d}} \\
\mathrm{wt}^{-} \%\end{array}$ & $\begin{array}{c}\mathbf{O}^{\mathrm{c}, \mathrm{d}} \\
\mathrm{wt}^{-} \%\end{array}$ \\
\hline $\mathrm{C}^{\mathrm{e}}$ & 315 & 0.19 & 98.1 & 0.8 & 1.1 \\
$\mathrm{C}^{2} 5 \mathrm{O}_{2}$ & 735 & 0.28 & 97.7 & 0.7 & 1.6 \\
$\mathrm{C}_{4} \mathrm{O}_{2}$ & 945 & 0.37 & 97.4 & 0.7 & 1.9 \\
\hline
\end{tabular}

a. BET surface area determined as per [58].

b. Micropore pore volume = cumulative volume up to $2 \mathrm{~nm}$.

c. From elemental analyser. Oxygen $\%$ is by difference.

d. Uncertainties: $\pm 0.3 \%$ for $\mathrm{C} ; \pm 0.1 \%$ for $\mathrm{H} ; \pm 0.2 \%$ for $\mathrm{O}$.

e. SSA \& SPV are in line with those obtained elsewhere [59].

\subsection{Characterization of carbon skeleton}

The carbonaceous skeleton of the three carbons along the activation pathway were each characterized by four methods, details of which follow below.

\subsubsection{Synchrotron-based XRD analysis}

X-ray powder diffraction experiments were performed at the Australian Synchrotron Powder Diffraction beam line, which is equipped with a Mythen II detector [60]. Samples were packed tightly into $0.3 \mathrm{~mm}$ diameter boron-rich glass capillaries (Charles Supper, USA) and then mounted on a rotational sample holder. The wavelength of the incident X-ray was determined to be $21 \mathrm{keV}$ ( $\lambda=0.059 \mathrm{~nm}$ ) by analysing the diffraction pattern of a NIST standard $\mathrm{LaB}_{6}$ sample. More detailed instrumental information can be found elsewhere [61].

The raw data for all the carbons and their corresponding empty capillaries were collected in the terms of intensity, $I$, versus scattering angle for the range $0.5^{\circ}<2 \theta<120^{\circ}$; each pattern was obtained using an exposure period of $1800 \mathrm{~s}$. This raw data was then processed using the PDFget X2 software [62]. Firstly, the pattern for the blank 
capillary was subtracted as background from the carbon sample pattern. The carbon sample pattern was then corrected for sample absorption, multiple scattering, beam polarization, Compton scattering and Laue diffuse scattering [43], after which the corrected intensity was expressed as a function of scattering vector $k=2 \sin \theta / \lambda$.

The corrected scattering function of carbonaceous materials such as those investigated here is typically characterised by a number of broad peaks that relate to short-range structure. Some of these peaks have long been used to characterise the carbon structure. Here, the average spacing between graphenic layers was estimated using the Bragg equation

$d_{002}=\frac{\lambda}{2 \sin \theta_{002}}=\frac{1}{k_{002}}$

where $k_{002}$ is the wave vector magnitude associated with the 002 peak, which can be determined by first subtracting the small angle scattering intensity from the spectra and then fitting a Gaussian function to the peak.

Whilst we recognize the questionable nature of the graphitic crystallite model [2], we have also used the 10 and 002 peaks in the corrected intensity function to obtain the average in-plane extent, $L_{a}$, and height, $L_{c}$, of the crystallites by using the Scherrer equation [2]

$L_{i}=\frac{K_{i} \lambda}{B_{i} \cos \theta_{i}}$

where $\theta_{i}$ and $B_{i}$ are the scattering angles and full width at half maximum (FWHM) values for the peaks, respectively, and $K_{a}=1.84$ and $K_{c}=0.89$.
In RDF analysis, $\rho(r)$, which gives the atomic number density at a radius $r$ from a central atom, was evaluated using [43]

$\rho(r)=\rho_{0}+\frac{G(r)}{4 \pi r}$

where $\rho_{0}$ is the average atomic number density of the scattering volume, which is well estimated by the carbon skeleton density for the range of $\rho(r)$ considered here (i.e. for $r<4 \AA$ ), and $G(r)$ is the reduced pair distribution function (PDF) obtained via [43]

$G(r)=8 \pi \int_{k_{\min }}^{k_{\max }} k[S(k)-1] \sin (2 \pi k r) d k$

where $\mathrm{S}(k)$ is the total structure factor that can be obtained from the corrected intensity function [43], and $k_{\max }$ and $k_{\min }$ correspond to the two extremes of the $2 \theta$ range probed by XRD.

The first of the peaks in the $\rho(r)$ is related to the atoms that are the nearest neighbors to the central atom. The average number of such nearestneighbor atoms was obtained from the area under this peak, which is given by [43]

$N_{1}=\int 4 \pi r^{2} \rho(r) d r$

where the integration limits are defined from the $G(r)[43]$.

The angle subtended by the two bonds connecting the central, first and second neighbouring atoms, $\varphi$, was obtained from the simple geometric relationship

$B_{23}=2 B_{12} \sin \left(\frac{\varphi}{2}\right)$

where $B_{12}$ is the bond length between the first and second atoms, and $B_{23}$ its counterpart between the second and third atoms, which can be estimated 
by the radial positions of the first and second peaks in the $\rho(r)$ respectively.

\subsubsection{Carbon skeleton and bulk densities}

A number of techniques have been used in the past to estimate the solid skeleton density, $\rho_{s}$, of nanoporous carbons, including mercury porosimetry [63] (e.g. [64]), He pycnometry [63] (e.g. [13]), analysis of the small-r region of the PDF [43] (e.g. [65]), and from small-angle X-ray scattering (SAXS) [66, 67]. The first of these approaches is unsuitable as it cannot probe the micropores that dominate the PFA-based carbons considered here [63]. The third approach is also unsuitable due to the limited $k$ range used here, which lead to excessive termination ripples at small-r [43] (indeed, even Dmowski et al. [65] observed significant uncertainty in the skeleton density of a nanoporous carbon when using this approach despite their scattering vector range being some 2.75 times greater than that used here). Whilst SAXS, unlike all the other methods, has the advantage of including the inaccessible porosity in the density evaluation, it was not readily available to us. Helium pycnometry was, therefore, used to determine the carbon skeleton density. The pycnometry was undertaken using helium at $40^{\circ} \mathrm{C}$. The pressure measurements were undertaken using a BELSORP-max gas sorption analyser (BEL, Japan). Especial care was made to ensure the system and sample temperature remained constant during the pycnometry analysis, as this can be a significant source of error. The densities for each carbon sample and the associated uncertainty were determined using results from a dozen repeat measurements for each carbon. The uncertainty in the densities is estimated to be $\pm 0.01 \mathrm{~g} / \mathrm{cm}^{3}$, equal to \pm 0.5 atoms $/ \mathrm{nm}^{3}$ or around $\pm 0.5 \%$ relative error.

The bulk density, $\rho_{b}$, of the carbon materials were estimated here using the expression [63]

$V_{p}=\frac{1}{\rho_{b}}-\frac{1}{\rho_{s}}$

where the micropore volume, $V_{p}$, was assumed to be equal to that determined using the nitrogen loading at $77 \mathrm{~K}$ and $P / P_{0}=0.995$ and a liquid density equal to the bulk density, $0.808 \mathrm{~g} / \mathrm{cm}^{3}$.

\subsubsection{EELS-SI}

The EELS-SI technique and the results it produces are illustrated in Fig. 2. Briefly, spatially resolved EEL spectra are obtained from the sample by scanning a focused sub-nanometre probe over a selected nanoscale area in nanometer-sized steps as shown in Fig. 2(a)-(c). As seen in Fig. 2(c)-(d), a map of the $s p^{2}$ fraction across this sample area can then be evaluated by comparison of its fine structure in the carbon Kedge core-loss spectrum with that of a reference material of known $s p^{2}$ content (r) using [68]

$f_{s p^{2}}=\left(\frac{I_{s}^{\pi^{*}}}{I_{s}^{\pi^{*}}+I_{s}^{\sigma^{*}}} / \frac{I_{r}^{\pi^{*}}}{I_{r}^{\pi^{*}}+I_{r}^{\sigma^{*}}}\right)$

where $I_{k}^{j}$ is the integrated intensity for the $j \in\left\{\pi^{*}, \sigma^{*}\right\}$ features, and the reference intensities used here were obtained from a highly-oriented pyrolytic graphite (HOPG) material (NT-MDT, Russia) known to contain only $s p^{2}$ carbon. 

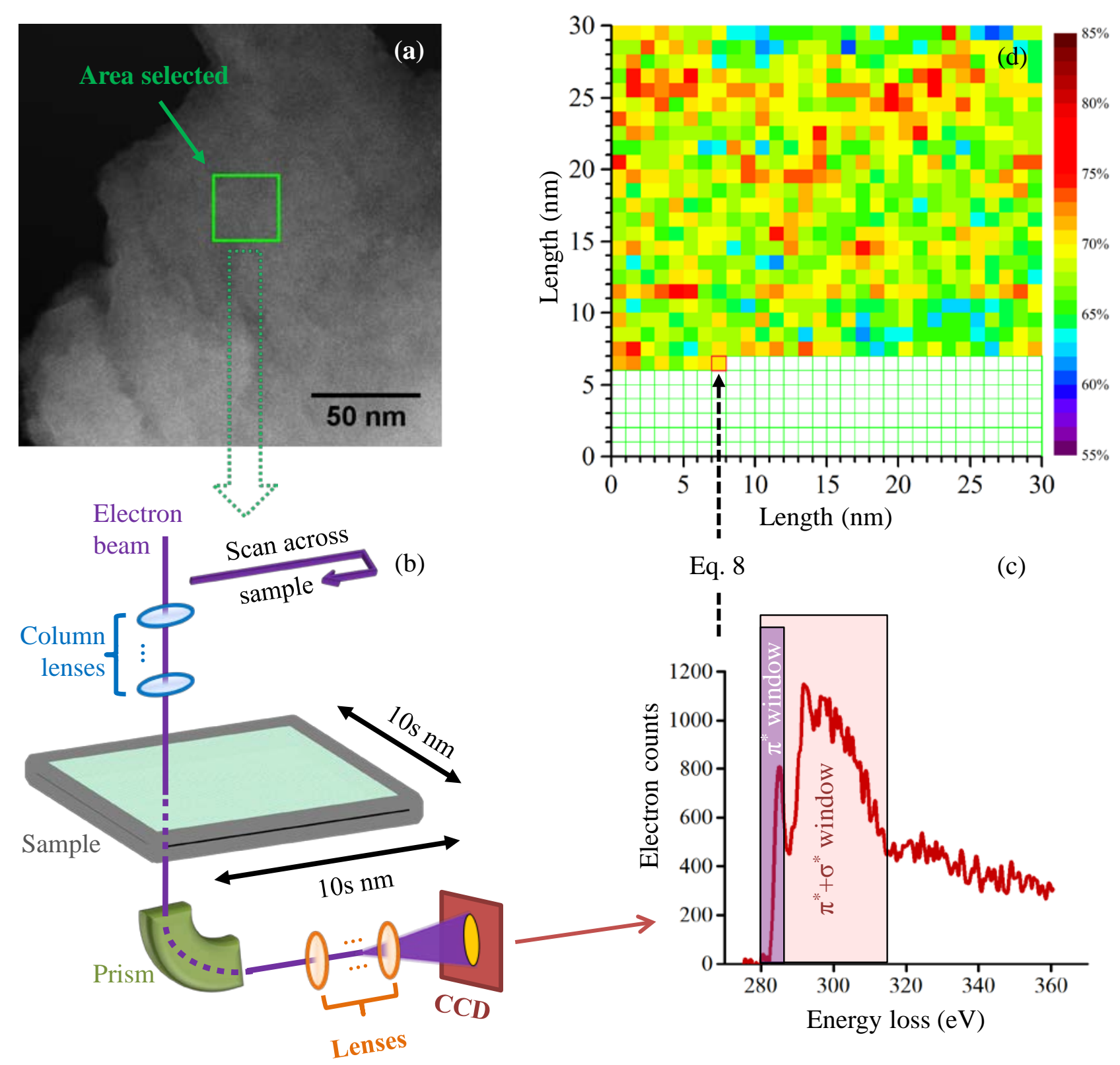

Fig. 2. Schematic of the EELS-SI technique and the results obtained from it: (a) an annular dark-field (ADF) STEM image is used to select an area for scanning; (b) a sub-nanometre focused electron beam is scanned in $\mathrm{O}(1) \mathrm{nm}$ steps across the selected area, with an individual EEL spectrum being collected at each step using an EEL spectrometer composed of an electron prism, lenses and charge-coupled-device (CCD) camera; (c) an individual spectrum from one step in the scan illustrating the integration windows for the $\pi^{*}$ and $\pi^{*}+\sigma^{*}$ regions; (d) the $100 \times f_{s p^{2}}$ (i.e. $\% s p^{2}$ ) map over the scanned area obtained using Eq. 8 with each individual spectrum.

There are two major approaches to determining the integrated intensities of the $\pi^{*}$ and $\sigma^{*}$ features in the carbon K-edge core-loss spectrum, namely: 2-window integration [52] and peak fitting [53].In the latter approach, it is important to ensure: (a) the peak positions and line shapes assigned have physical significance; (b) the fitting routine is robust for all samples; and (c) the final fitting residue is small. These criteria are in general difficult to fulfil for EEL spectra of nanoporous carbons because their $\pi^{*}$ and $\sigma^{*}$ features are shifted, broadened and asymmetric, 
and there are sometimes additional features that do not exist in the reference material such as those that can appear between 280-285 eV and around $290 \mathrm{eV}$ from carbon atoms at the edges of graphene layers [69], and between the $\pi^{*}$ and $\sigma^{*}$ peaks due to non-hexagonal rings [53]. To avoid these issues, we employ here the simpler direct integration method. The $\pi^{*}$ integration window is set to the energy range of the $\pi^{*}$ peak of HOPG, namely 280-286 eV, whilst that of the whole carbon edge was chosen to be $35 \mathrm{eV}$ from the onset of the edge (i.e. 280-315 eV). Use of these two windows in evaluating Eq. 8 should yield a $s p^{2}$ fraction that excludes those carbon atoms that reside in 5- and 7-membered rings and at the edge of graphene sheets.

In the work reported here, the EELS-SI characterisation was carried out in a dualcorrected FEI Titan ${ }^{3}$ 80-300 FEGTEM fitted with CEOS aberration correctors on both illumination and imaging lenses. The TEM was operated at 80 $\mathrm{keV}$; based on a theoretical calculation of electron knock-on damage of carbonaceous materials [70], sample damage would be negligible under such a voltage. Areas of $30 \times 30 \mathrm{~nm}^{2}$ in size were scanned across by a $1 \mathrm{~nm}$ FWHM electron probe in $1 \mathrm{~nm}$ steps, giving a total of 900 spatially resolved spectra per sample. The spectra were collected using a Gatan Tridiem 863P image filter in spectroscopy mode (the EEL spectrometer in Fig. 2). Magic angle conditions (convergence semiangle $\alpha=2.7 \mathrm{mrad}$ and collection semi-angle $\beta=$ $2.7 \mathrm{mrad}$ ) were employed to suppress changes to the fine structure in the carbon K-edge due to the anisotropic graphitic structure and allow accurate $s p^{2}$ fraction estimation [71]. The spectra resolution was determined to be around $0.7 \mathrm{eV}$ by measuring the FWHM of the zero-loss peak collected in vacuum (no sample exposed to the electron probe). The background was subtracted from each carbon K-edge core-loss spectrum by using a fitted power-law distribution function. Further multiple scattering effects were removed by using the Fourier ratio method [72].

\subsubsection{Nanodiffraction and FEM}

The FEM technique in STEM mode [73] and the results it produces are illustrated in Fig. 3. Briefly, spatially resolved electron diffraction patterns are obtained for the sample by scanning a nanometresized focused probe over a selected nanoscale area in sub-nanometer sized steps as illustrated in Fig. 3(a)-(b). Each diffraction pattern reflects the atomic ordering of the skeleton within the nanometre-sized domain probed, Fig. 3(c). As shown in Fig. 3(d), these nanodiffraction patterns for the selected area can be averaged over to yield an average diffracted intensity $\langle I(\mathbf{k})\rangle$ with slightly converged illumination (1.3 mrad), where $I$ is the intensity, and $\mathbf{k}$ is the scattering vector. This can in turn be used as a basis for evaluating the normalized intensity variance, $V(\mathbf{k})$, as per [45]

$V(\mathbf{k})=\left(\left\langle I^{2}(\mathbf{k})\right\rangle-\langle I(\mathbf{k})\rangle^{2}\right) /\langle I(\mathbf{k})\rangle^{2}$

This gives the variation in diffracted intensity into each scattering vector from area to area. Finally, both the average diffracted intensity and normalized intensity variance can be azimuthally averaged to yield their one-dimensional counterparts. 


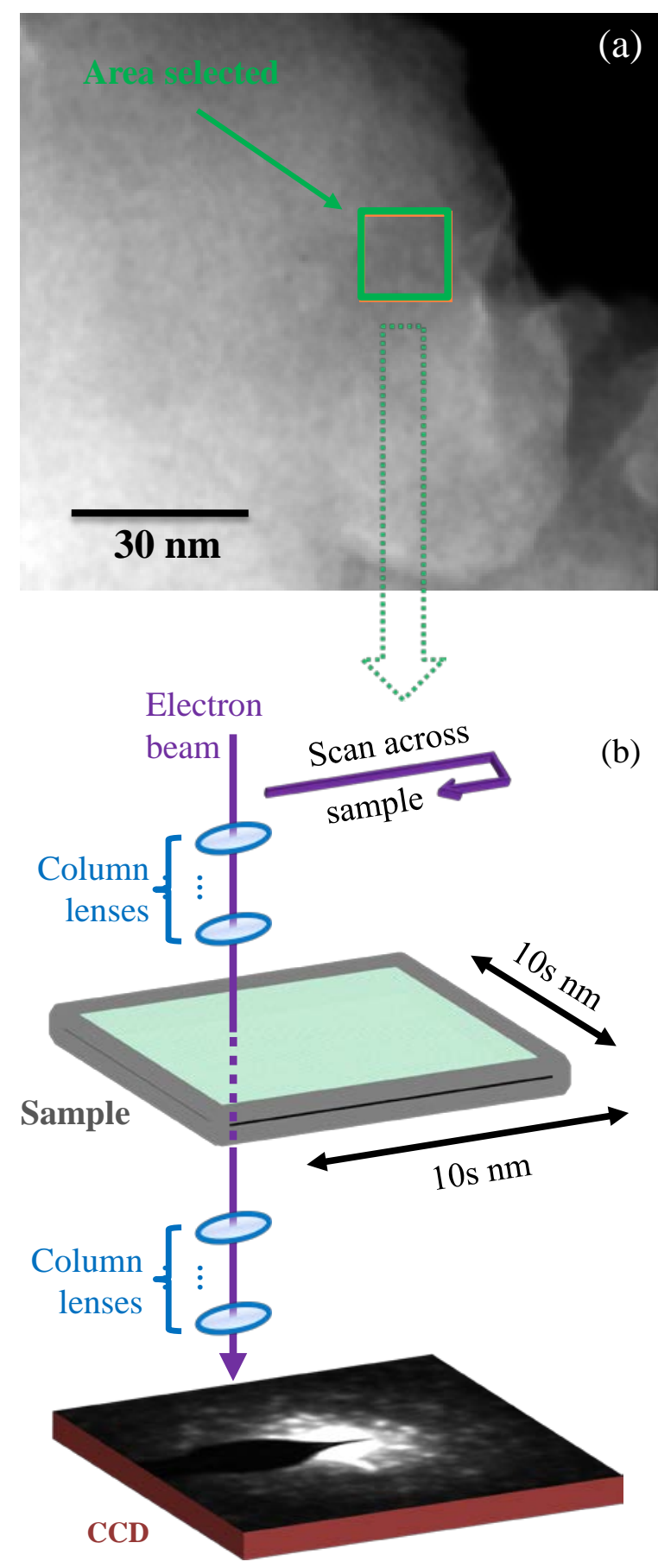

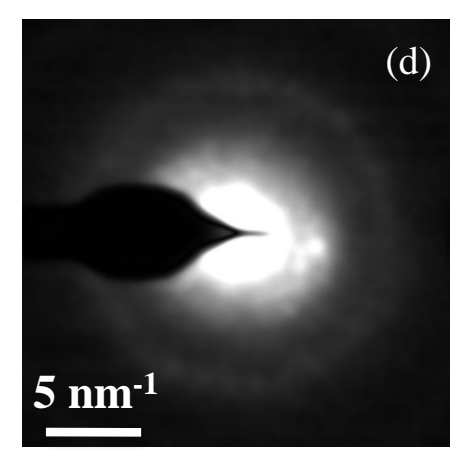

Averaged intensity

for selected area

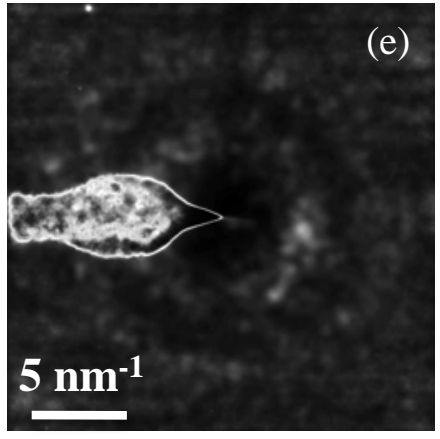

Normalized intensity variance for selected area

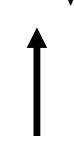

Average over 100s-1000s of patterns

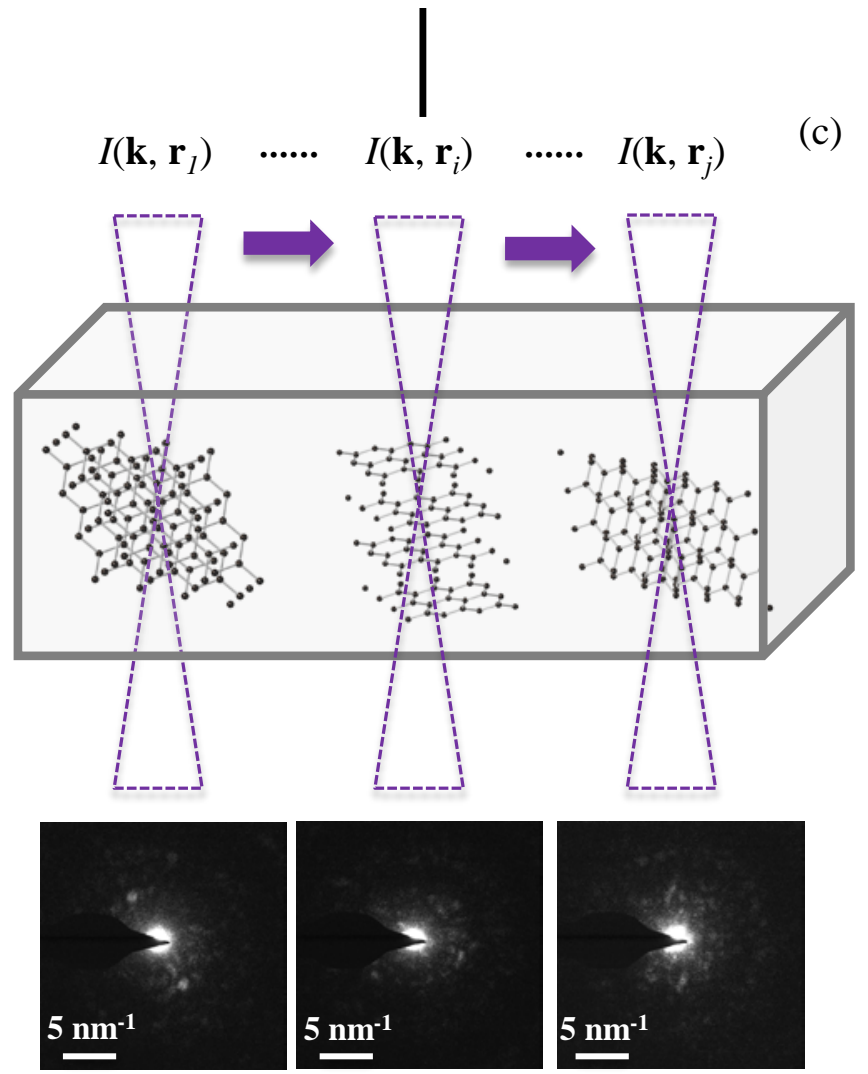

Fig. 3. Schematic of the FEM technique and the results obtained from it: (a) An ADF image is used to select an area for scanning. (b) An $\mathrm{O}(1) \mathrm{nm}$ focused electron beam is scanned in sub-nanometre steps across this area, with an individual electron diffraction (nanodiffraction) pattern being collected at each step using a charge-coupled device (CCD) camera. (c) The individual nanodiffraction patterns for each position, $\mathbf{r}$, reflect the skeleton structure within the nanoscale volume probed. (d) The electron diffraction and normalised intensity variance can be obtained for the area by averaging over all nanodiffraction patterns.

FEM was performed here in the STEM mode on the same instrument and at the same energy as the EELS-SI experiments. The electron probe convergence angle was 1.3 mrad resulting in an aperture-limited probe with a FWHM of $2.4 \mathrm{~nm}$. Four areas each of $15 \times 15 \mathrm{~nm}^{2}$ size were scanned with the electron probe in $0.5 \mathrm{~nm}$ steps, giving a total of $4 \times 900$ spatially resolved nanodiffraction 
patterns per sample. The regions examined using FEM were selected to ensure the thicknesses of the areas were all roughly constant at approximately 20-30 nm, which was estimated using the low loss regions of the EEL spectra. The nanodiffraction patterns were recorded on a Gatan UltraScan 1000 P $(2 \mathrm{k} \times 2 \mathrm{k})$ CCD camera using an exposure time of 0.5 s. The nanodiffraction patterns were treated with a lowpass Butterworth filter of order two to minimize noise. The relative error in the measured normalized intensity variance due to Poisson noise in the individual diffraction patterns was below 0.02 [73]. The error in the normalized intensity variance was calculated as the standard error from the four scanned areas.

\section{Results and Discussion}

\subsection{Carbon skeleton density}

The carbon skeleton and bulk densities for the three carbon materials, $\rho_{s}$ and $\rho_{b}$ respectively, are given in Table 2 . The skeleton density for the C0 material is consistent with that obtained previously by others for a PFA-based carbon obtained using a carbonization temperature of $800^{\circ} \mathrm{C}$ [13]. The increasing trend of the skeleton density with conversion seen here is in line with that observed by Burket et al. [17]. Although the density of their unactivated material is around $10 \%$ greater than here, likely due to the different preparation conditions, the increase they saw thereafter is roughly commensurate with what was seen here: $8 \%$ up to $25 \%$ conversion here compared to their $10 \%$, and $16 \%$ up to $45 \%$ conversion for both studies. The approach of the skeleton density to $~ 93 \%$ that of graphite (110 atoms $/ \mathrm{nm}^{3}$ ) is also in line with the observations of Burket et al. [17]. Given that at $45 \%$ conversion the carbon is unlikely to contain significant closed porosity, this provides some support for the notion that the skeleton has experienced densification during oxidation.

The bulk densities are smaller than the skeleton density, especially in the activated materials, commensurate with their highly porous nature. The decrease in the bulk density with conversion is, of course, in line with the increase in porosity and skeleton density as per Eq. 7.

Table 2. Carbon skeleton density, $\rho_{s}$, and bulk density, $\rho_{b}$, of the three carbons studied here.

\begin{tabular}{ccc}
\hline Carbon & $\boldsymbol{\rho}_{\boldsymbol{s}}\left(\mathbf{a t o m s} / \mathbf{n m}^{\mathbf{3}}\right)^{\mathbf{a}}$ & $\boldsymbol{\rho}_{\boldsymbol{b}}\left(\mathbf{a t o m s} / \mathbf{n m}^{\mathbf{3}}\right)^{\mathbf{b}}$ \\
\hline $\mathrm{C} 0$ & 86 & 65 \\
$\mathrm{C} 25 \mathrm{O}_{2}$ & 93 & 61 \\
$\mathrm{C}_{4} 5 \mathrm{O}_{2}$ & 102 & 58 \\
\hline
\end{tabular}

a. Uncertainty is \pm 0.5 atoms $/ \mathrm{nm}^{3}$.

b. Uncertainty is \pm 1 atoms $/ \mathrm{nm}^{3}$.

\subsection{XRD analysis}

Fig. 4(a) shows the corrected intensity function for the three carbon samples up to $k=20 \mathrm{~nm}^{-1}$, which is the range within which features are discernable. The 10 and 11 graphitic peaks are clearly resolved for all the carbons, and there is some evidence of 20 and 21 graphitic peaks as well as two further broad peaks beyond these (indicated by arrows) that correspond to compound graphitic peaks. At the very least these peaks collectively suggest a short-range graphenic structure holds along the activation pathway. The 002 graphitic peak is also very well resolved for the three carbons, suggesting short- 
range graphitic stacking is also present along the activation pathway. There is additionally some asymmetry on the right-hand side of the 10 peak and, to an even lesser extent, the 11 peak. Whilst these features could arise for a number of reasons, they could possibly be evidence of 004 and 006 graphitic peaks, suggesting some presence of graphitic stacking beyond the minimum indicated by the strong 002 peak.
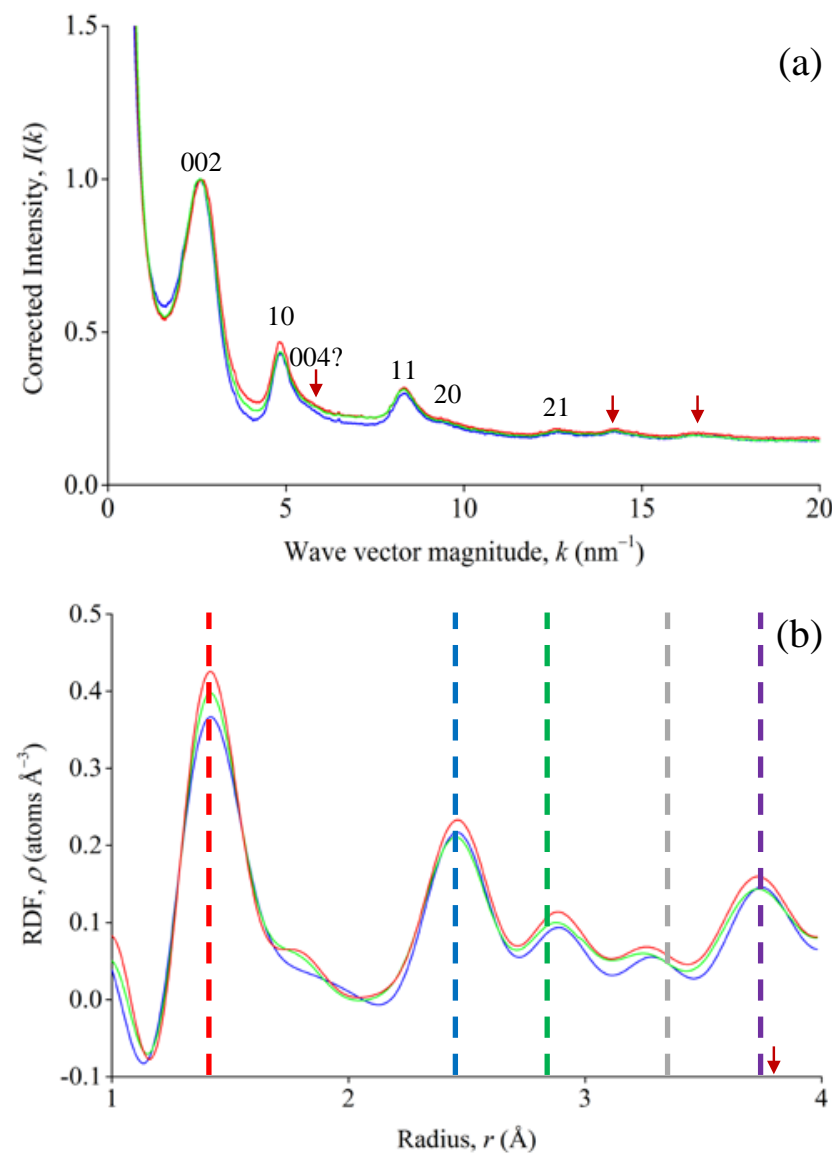

Fig. 4. XRD-derived functions for the $\mathrm{C} 0$ (solid blue line), $\mathrm{C}_{25 \mathrm{O}}$ (solid green line) and $\mathrm{C}_{4} \mathrm{O}_{2}$ (solid red line) carbons: (a) corrected intensity function with Bragg reflections indicated where discernable; and (b) $\rho(r)$ along with the expected positions of the carbon atoms in a graphene sheet shown by vertical coloured dashed lines where the colours correspond to those used in Fig. 5.

Table 3 details the average inter-layer spacing and graphitic crystallite dimensions obtained using Eq. 1 and Eq. 2 respectively. This table shows that the spacing between commensurate graphene layers is greater than the $0.336 \mathrm{~nm}$ of graphite, in line with the 002 peak being shifted to a lower scattering vector compared to that of graphite. It, however, also suggests that both this spacing and the graphitic crystallite sizes remain unchanged during activation. This is contrary to the skeleton density data reported above and, as will be seen below, the RDF, EELS-SI and FEM analysis.

Table 3. Carbon skeleton parameters derived from Bragg and Scherrer analysis, Eq. 1 and Eq. 2 , of the corrected intensity function.

\begin{tabular}{cccc}
\hline Carbon & $\boldsymbol{d}_{\mathbf{0 0 2}}{ }^{\mathbf{a}}(\mathbf{n m})$ & $\boldsymbol{L}_{\boldsymbol{a}}^{\mathbf{b}}(\mathbf{n m})$ & $\boldsymbol{L}_{\boldsymbol{c}}^{\mathbf{b}, \mathbf{c}} \mathbf{( n m )}$ \\
\hline $\mathrm{C} 0$ & 0.38 & 2.9 & 0.9 \\
$\mathrm{C}^{2} 5 \mathrm{O}_{2}$ & 0.38 & 3.1 & 0.9 \\
$\mathrm{C}_{4} \mathrm{O}_{2}$ & 0.37 & 3.1 & 0.9 \\
\hline
\end{tabular}

a. Uncertainty is $\pm 0.01 \mathrm{~nm}$.

b. Uncertainty is $\pm 0.1 \mathrm{~nm}$.

c. Parallelism indicator [74] is also constant at $\boldsymbol{R}=2.38$.

Fig. 4(b) shows the $\rho(r)$, which is consistent with that obtained by others for nanoporous carbons from various precursors and synthesis methods $[13,65,75-77]$. Comparison of the positions of all but the fourth peak with the short-range graphenic structure, Fig. 5(a), confirms the conclusions drawn from qualitative analysis of the corrected intensity function. The broadness of the peaks along with the fourth peak and shoulder to the right of the first peak are indicative of deviations from graphene, which we will explore in more detail in the following.

All three carbons possess a peak in the $\rho(r)$ that is centred around $1.42 \AA$, which is commensurate with the $\mathrm{C}-\mathrm{C}$ bond in a six-membered ring as illustrated in Fig. 5(a). The nearest neighbour coordination number, $N_{1}$, obtained from applying Eq. 5 to this peak is given in Table 4 for the 

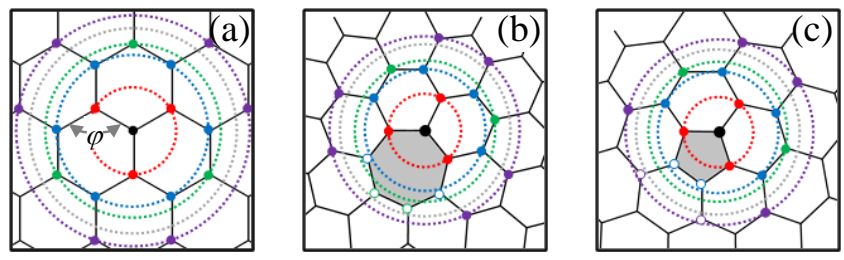

Fig. 5. Graphenic sheets showing the $1^{\text {st }}(\mathrm{red}), 2^{\text {nd }}$ (blue), $3^{\text {rd }}$ (green) and $4^{\text {th }}$ (violet) nearest neighbouring rings of carbon atoms around a central carbon atom (black dot) along with a ring that corresponds to the radius of the fourth peak in Fig. 4(b) (grey): (a) perfect graphene sheet; (b) sheet containing a heptagon defect; and (c) sheet containing a pentagon defect. The ring colours correspond to the vertical lines in Fig. 4(b). The hollow dots in (b) \& (c) represent atoms whose distance from central atom is altered due to the defect. Structures in (b) \& (c) are those predicted by Yazyev \& Louie [78] using an $a b$ initio method.

Table 4. Carbon skeleton parameters derived from analysis of the first two peaks of the $\rho(r)$.

\begin{tabular}{cccc}
\hline Carbon & $\boldsymbol{N}_{\mathbf{1}}{ }^{\mathbf{a}}$ (bonds) & $\mathbf{F W H M}_{\mathbf{1}}{ }^{\mathbf{b}}(\mathbf{\AA})$ & $\boldsymbol{\varphi ( { } ^ { \circ } ) ^ { \mathbf { c } }}$ \\
\hline $\mathrm{C} 0$ & 2.72 & 0.26 & $103-146$ \\
$\mathrm{C}^{2} 5 \mathrm{O}_{2}$ & 2.84 & 0.24 & $103-146$ \\
$\mathrm{C}_{5} \mathrm{O}_{2}$ & 2.91 & 0.22 & $103-146$ \\
\hline
\end{tabular}

a. From $\rho(r)$ via Eq. 5 ; uncertainty is \pm 0.6 bonds.

b. Uncertainty is $\pm 0.2 \AA$.

c. From $\rho(r)$ via Eq. 7.

carbons, where the error introduced by the definition of the integration limits is estimated to be \pm 0.06 . The value for the $\mathrm{C} 0$ carbon is in line with that obtained by Petkov et al. [13] for a similar unactivated material. The nearestneighbour coordination number for perfect graphene of infinite size is 3 . A value below this is indicative of possible in-plane vacancies and edge atoms. As in-plane vacancies are likely to be more reactive sites compared to other atoms and be healed due to carbon atom migration at elevated temperatures [79], they are unlikely to be too numerous in the carbons here compared to the edge site density. We, therefore, ascribe the reduction of $N_{1}$ below the ideal limit to edge sites. This allows an estimate of some 'average' size of the graphene sheets in the three carbons to be made. As Fig. 6 indicates, the increasing nearestneighbour coordination number along the activation pathway is indicative of a corresponding increase in the in-plane coherence length, with this length nearly doubling up to $25 \%$ conversion, and increasing nearly as much again up to $45 \%$ conversion. Whilst the structures and sizes in Fig. 6 are only illustrative and will clearly be affected by the presence of defects such as those in Fig. 5(b) \& (c) and the curvature they introduce, it should be noted that the carbon atoms associated with such defects are still bonded with three neighbouring atoms and, thus, will not affect the value of $N_{1}$.

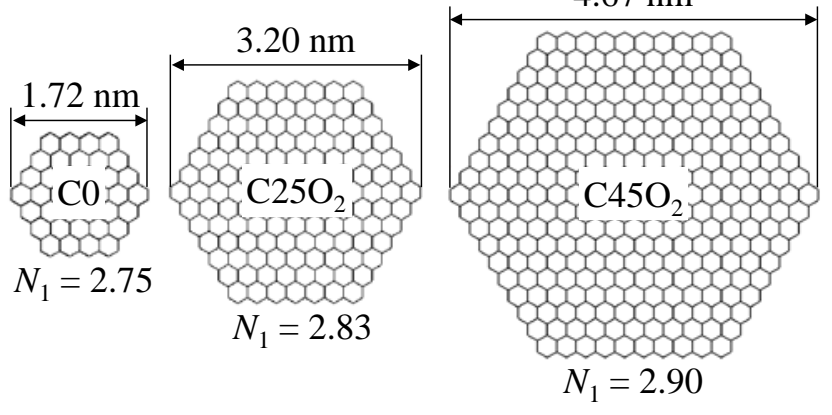

Fig. 6. Finite size perfect graphene layers that match the nearest-neighbour coordination number, $N_{1}$, for the three carbons (see Table 4).

The broadness of the first peak in Fig. 4(b), which is shown in Table 4 in the form of its FWHM, is some 2-3 times that of HOPG [65]. This indicates the presence of some non-graphenic (i.e. defective) carbon. The uncertainty in the FWHM is, unfortunately, too great to claim anything definitive from its reduction with activation. However, it possibly suggests the amount of non- 
graphenic carbon is being reduced by activation, something we will return to further below when considering the results from the EELS-SI analysis. Moving beyond the first peak in the $\rho(r)$, comparison of Fig. 4(b) with Fig. 5(a) indicates the second, third and fifth peaks can be confidently attributed to carbon atoms within a graphenic layer. Whilst the position and widths of these peaks remain essentially invariant throughout activation, their increase in height suggests the local in-plane structure is becoming denser as activation proceeds.

Assuming the widths of these two peaks reflect, in part, distortion of the graphenic structure, Table 4 shows that this angle takes on a range of values that is invariant with activation and in line with those obtained from Reverse Monte Carlo simulation of nanoporous carbons [80-82].

Of all the peaks that are clearly related to the graphenic structure, the third is most distorted from that expected for a perfect graphene sheet, with its peak being around $0.05 \AA$ higher than the ideal. This cannot be linked to in-plane defects such as those shown in Fig. 5(b) and (c), which push carbon atoms into positions between the third and fourth and second and third peaks respectively. However, the third coordination shell in diamond is located around $0.8 \AA$ above the position of the maximum of the third peak (i.e. at $2.96 \AA$ ), which means the maximum is 'bracketed' by this and the corresponding coordination shell in graphene. This may suggest the possible existence of some $\mathrm{sp}^{3}$ carbon atoms, although the clear absence of any other diamond- related peaks, which would be located at $1.54 \AA$, $2.52 \AA, \quad 3.56 \AA$ and $3.88 \AA$, indicates the concentration of such atoms is substantially lower than the $s p^{2}$ carbon.

Two other peaks occur in the $\rho(r)$ of Fig. 4(b) that do not correspond well to the perfect graphenic structure. The first is the shoulder on the first peak at around $1.8 \AA$, whilst the second is a distinct but small peak at $3.3 \AA$. A number of other groups report similar features for carbon materials, including activated carbons [65, 83, 84], non-graphitizing carbons $[13,65]$ and even coals [85]. Interestingly, the first of these features is absent from the $\mathrm{C} 0$ carbon but develops during activation. This suggests it may have something to do with the introduction of heteroatoms, most likely oxygen, whose concentration rises significantly during activation as shown in Table 1. Moving on, although the fourth peak in the $\rho(r)$ is located around that of the interlayer spacing of graphite (3.36 $\AA$ ), it is highly unlikely to be due to such layering given the disordered nature of the carbon. Instead we propose this peak most likely arises from the presence of heptagon defects like that shown in Fig. 5(b) whose outer carbon atoms (shown as open green circles) are located at $\sim 3.27 \AA$ from the central atom [78]. This interpretation needs to be treated with caution, however, as there is no obvious evidence in the $\rho(r)$ of the next innermost atoms in the heptagon (shown as open blue circles) nor the significant pushing out of the fourth coordination shell, although both could be hidden in the upper reaches of the second and fifth peaks respectively (at $\sim 2.65 \AA$ and $\sim 3.9 \AA$ ) given the concentration 
of such defects will be small based on the size of the fourth peak.

If heptagons are present within the graphenic structure, one might expect pentagons to also exist. As Fig. 5(c) indicates, this would lead to carbon atoms existing somewhat inside the second coordination shell associated with perfect graphene at $\sim 1.9 \AA$ (shown as open blue circles), and slightly inside the fourth shell (shown as open violet circles) that is itself pushed outwards significantly (i.e. at $\sim 3.7 \AA$ ) [78]. It is, however, difficult to discern any evidence for these atoms as the first position is at the upper end of the small feature on the shoulder of the first peak (i.e. it is unlikely to be the main contributor to this feature, but may be a minor part of it), whilst the second is firmly in the body of the fifth peak.

\subsection{EELS-SI}

Fig. 7 shows the carbon K-edge spectra averaged over the $30 \times 30 \mathrm{~nm}^{2}$ area scanned for each of the carbons in addition to the HOPG. The spectra of the three carbon samples possess four major features within the 270-360 eV energy loss range.

The first feature is the well-defined peak centered at $285 \mathrm{eV}$ that is due to the transition of the $1 \mathrm{~s}$ electron to the $\pi^{*}$ anti-bonding orbital. As suggested by its dominance in the normalized HOPG spectrum, Fig. 7(b), this transition is characteristic of $s p^{2}$ carbon. Whilst this peak for the three carbon samples is less prominent compared to HOPG, it still very clearly exists, with its relative intensity increasing along the activation pathway, particularly in the initial activation phase (i.e. from $\mathrm{C} 0$ to $\mathrm{C}_{25} \mathrm{O}_{2}$ ).
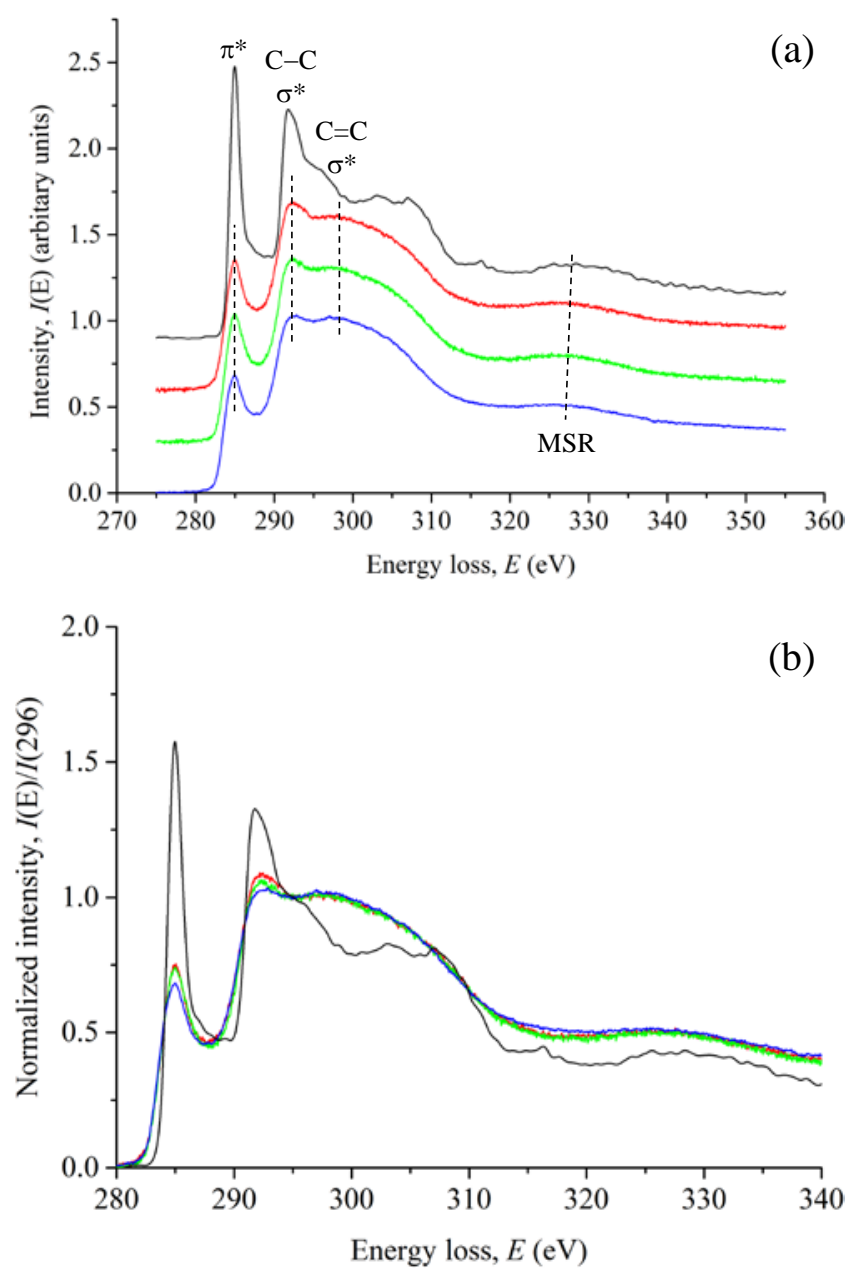

Fig. 7. Average EELS core loss spectra for the $\mathrm{C} 0$ (blue line), $\mathrm{C}_{25 \mathrm{O}}$ (green line) and $\mathrm{C}_{45 \mathrm{O}}$ (red line) carbons, and HOPG (black line): (a) Raw spectra shifted to facilitate qualitative comparison of features; and (b) normalised spectra to facilitate quantitative comparison of features. The spectrum for each material is an average of the 900 spectra obtained from scanning a $30 \times 30 \mathrm{~nm}^{2}$ area of the material.

The second major feature in the spectra, which is centred at around $292 \mathrm{eV}$, is associated with the $1 \mathrm{~s}-\sigma^{*}$ excitation, with Zhang et al. [53] specifically linking it to the $\mathrm{C}-\mathrm{C}$ carbons. Inspection of the normalised spectra shows that whilst it is a weak feature in the unactivated char, it grows steadily along the activation pathway into a distinct peak, in line with observations for graphitization under inert environments [53]. A similar development of this peak was also 
correlated with increased graphitization in TiCbased carbons [86].

The penultimate major feature in the spectra is the broad hump that roughly spans the energy loss range of 295-315 eV. This feature is not immediately apparent in the normalized HOPG spectrum, Fig. 7(b), although it may possibly be related to some of the fine features that appear in the graphite spectrum in this energy range. Based on carbon K-ionization edge spectra for gaseous molecules, Zhang et al. [53] specifically link this feature to the $1 \mathrm{~s}-\sigma^{*}$ excitation for $\mathrm{C}=\mathrm{C}$ carbons. This interpretation should, however, be viewed with some caution as Fig. 7(b) shows that, unlike its much narrower C-C counterpart and the $\pi^{*}$ peak, it does not change markedly along the activation pathway.

The final feature in the EELS spectra is the broad peak centered at around $328 \mathrm{eV}$, which others [52] have associated with multiple scattering resonance (MSR). It has been argued that the position of this is inversely related to the C-C bond length [52] and, as such, any difference in the energy value is indicative of a difference in the bond length. On this basis, as the MSR feature for the HOPG is up-shifted relative to those of the three carbon samples, Fig. 7 suggests not unsurprisingly that the mean $\mathrm{C}-\mathrm{C}$ distance of all three carbons are greater than those of graphite. However, no statistical difference exists between the locations or widths of these features for the three carbons.

Fig. 8 shows the $s p^{2}$ fraction map obtained for the three carbons. Visual comparison of the maps in
Fig. 8(a) and Fig. 8(b) for two unrelated areas of the $\mathrm{C} 0$ carbon suggests the measurements for a material are repeatable. Statistical analysis [87] of the distributions of $s p^{2}$ fractions across the two areas, which are shown in Fig. 9(a), supports this notion, with the means and variances being statistically identical with $99.99 \%$ and $99 \%$ confidence respectively. The average $s p^{2}$ fractions obtained for the two areas of the un-activated carbon (C0), which are given in Table 5, are comparable with the fractions obtained for other carbons obtained at similar temperatures [52, 53].

Fig. 9(a) indicates that the carbonaceous skeleton of the unactivated char contains a substantial fraction of non-sp $p^{2}$ bonded carbons - 20\% on average with some nanoscale volumes as high as $\sim 30 \%$. The significant absence of spatial correlations in the maps of Fig. 8(a)-(b) also show that the skeleton is poorly organised on the nanoscale. This suggests that some of the disorder inherent to the PFA precursor [88] is still retained through the carbonization process used here, in line with the conclusions drawn some time ago [10] using different experimental methods.

Table 5. Gaussian fits to histograms in Fig. 9.

\begin{tabular}{cccc}
\hline Carbon & Mean (\%) & FWHM (\%) & $\boldsymbol{R}^{\mathbf{a}}$ \\
\hline C0 area 1 & $78.45(0.11)$ & $7.79(0.27)$ & 0.95 \\
C0 area 2 & $78.75(0.09)$ & $7.97(0.09)$ & 0.97 \\
$\mathrm{C}^{\mathbf{2 b}} \mathrm{O}_{2}$ & $79.46(0.09)$ & $8.51(0.26)$ & 0.97 \\
$\mathrm{C}^{\mathbf{b}} \mathrm{O}_{2}$ & $81.48(0.12)$ & $8.00(0.30)$ & 0.95 \\
\hline
\end{tabular}

a. Standard errors are shown in parentheses.

b. Coefficient of determination for fit to histogram.

Comparison of the $s p^{2}$ maps for the un-activated carbon with those of the $\mathrm{C}_{2} 5 \mathrm{O}_{2}$ and $\mathrm{C} 45 \mathrm{O}_{2}$ carbons, which are shown in Fig. 8(c) and (d) respectively, clearly shows that the fraction of $s p^{2}$ 

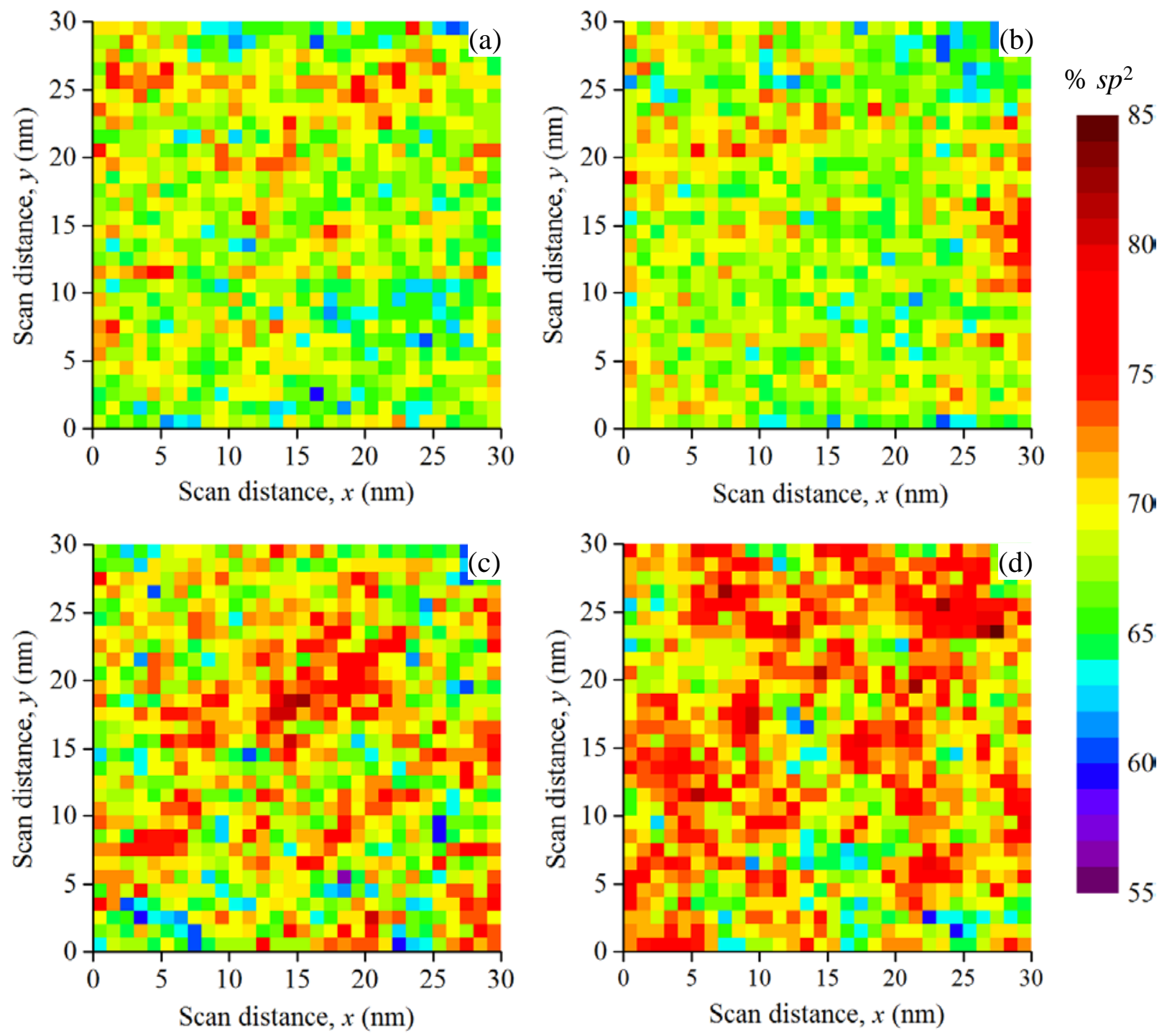

Fig. 8. Maps of percentage of carbon that is $s p^{2}$ hybridized as determined from applying EELS-SI to $30 \times 30 \mathrm{~nm}^{2}$ areas of carbon: (a) C0 (area 1); (b) C0 (area 2); (c) $\mathrm{C}_{25 O}$; and (d) $\mathrm{C}_{45 \mathrm{O}_{2}}$. Areas 1 and 2 of the $\mathrm{C} 0$ carbon are independent.
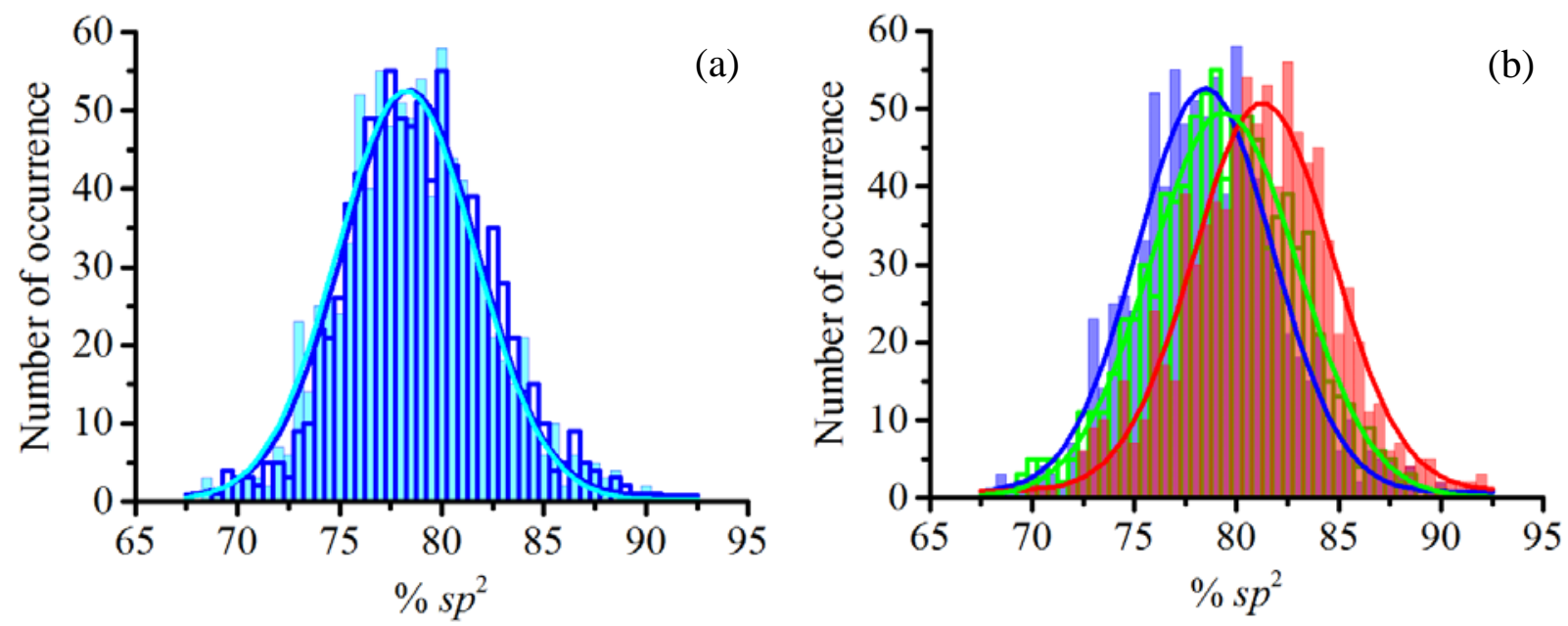

Fig. 9. Histograms of $\% s p^{2}$ hybridization derived from the maps in Fig. 8 (bars) with Gaussian fits (lines) for: (a) two independent areas of carbon C0; and (b) scanned areas of carbons C0 (blue), $\mathrm{C}_{25} \mathrm{O}_{2}$ (green) \& $\mathrm{C}_{45 \mathrm{O}}$ (red). Histograms are based on 900 data points except for C0 in (b), which is based on 1800 points. 
carbon and its spatial correlation increases along the activation pathway - in other words, the activation processes induces an increase in order at both the microscale and mesoscale. The former is confirmed in Fig. 9(b) and Table 5, which shows an up-shift in the $s p^{2}$ distribution along the activation pathway, albeit by only $~ 1 \%$ at $25 \%$ conversion accelerating to $3 \%$ at $45 \%$ conversion; statistical analysis [87] confirms these changes in the distributions are real with 99.99\% confidence.

\subsection{TEM, Nanodiffraction and FEM}

Fig. 10 shows example TEM images for the C0 and $\mathrm{C}_{45} \mathrm{O}_{2}$ samples. Both specimens present clear fringe patterns. In the unactivated material, the fringes are well-packed, suggesting the porosity is tight, in line with the picture presented by Mariwala and Foley [10] based on less-direct methods. Whilst caution should be exercised [44], assuming the fringes are the edges of graphene layers, the TEM image of the $\mathrm{C} 0$ carbon is suggestive of considerable curvature in the planes and their stacking (e.g. area in solid circle). Interestingly, the latter is contrary to the evidence presented so far in this report, emphasising the caution one must bring to interpreting TEM images of disordered carbon materials such as those being investigated here. The TEM image for the activated material, Fig, 10(b), also suggests considerable curvature (e.g. that pointed at by arrows) and stacking (e.g. area in solid circle). There is, however, also some evidence of more open areas (e.g. areas in broken circles) as one might expect given the extent of activation (45\% conversion).
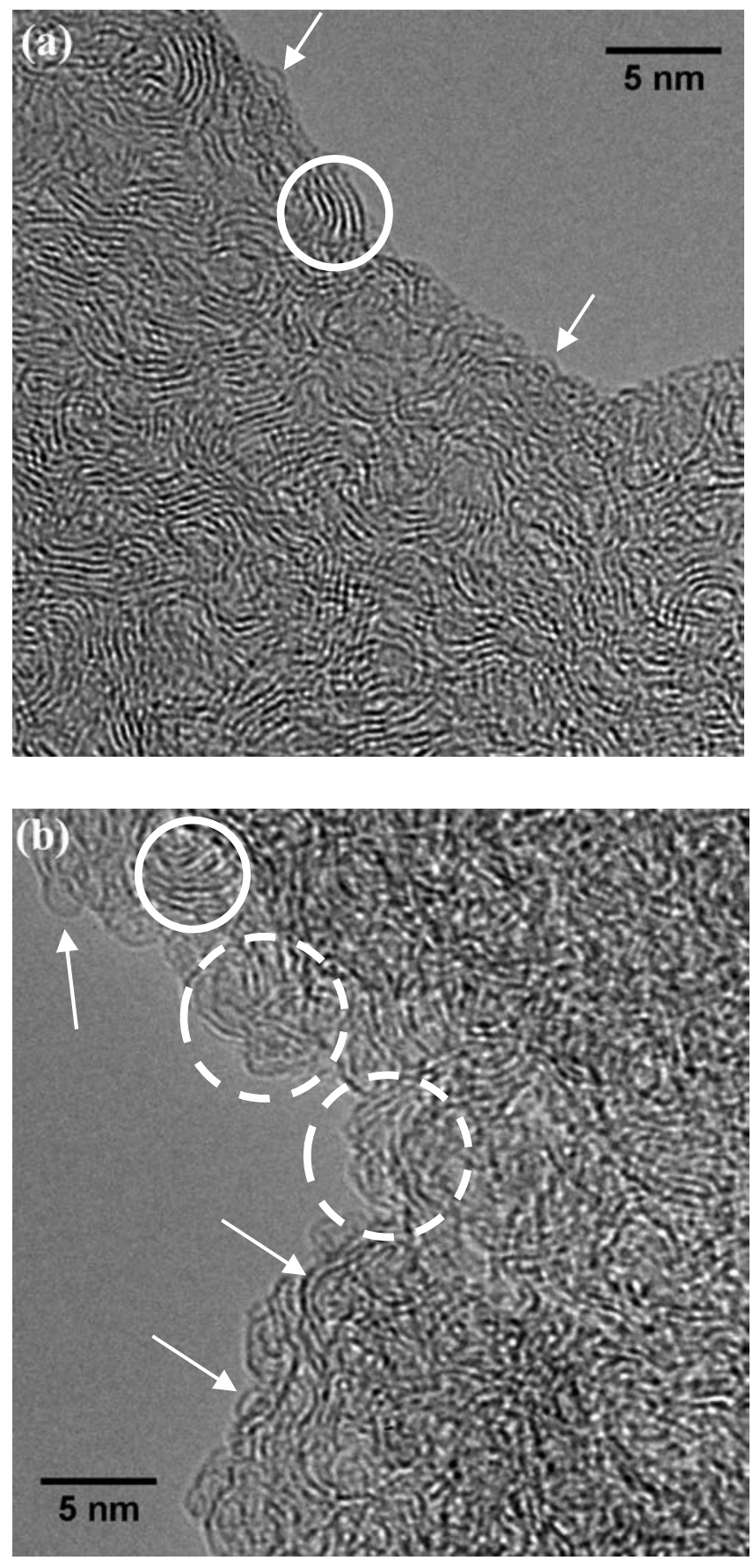

Fig. 10. Example TEM images of carbons: (a) C0; and (b) $\mathrm{C}_{45} \mathrm{O}_{2}$. See text for reference to marked features.

FEM can provide a more objective basis for confirming or rejecting the contention that microscale and mesoscale order is increasing along the activation pathway as suggested by the RDF and EELS-SI analysis. Fig. 11(a) shows for the $\mathrm{C} 0$ and $\mathrm{C}_{4} \mathrm{O}_{2}$ carbons the azimuthallyaveraged diffracted intensity derived from the 
average of the 3600 electron nanodiffraction patterns obtained from the selected scanned areas of both carbons, whilst Fig. 11(b) shows the associated normalized intensity variance, Eq. 9. Both curves in this figure contain two broad peaks. The first is located at around $4 \mathrm{~nm}^{-1}$, which corresponds to the 10 reflection, and the second is located at around $8 \mathrm{~nm}^{-1}$, which corresponds to the 11 reflection. Both reflections are due to intra-plane periodicities as shown in the inset of Fig. 11(a).

Although there is a small shoulder in the variance data at low $\mathrm{k}$ that may be assigned to the 002 reflection, the absence of a well-defined 002 peak is unexpected given its presence in the XRD pattern. This may arise from graphitic registry between layers being confined to finite-sized clusters that are sufficiently dispersed to not be detected in the areas scanned in the study here but which are of high enough concentration that they can be detected in bulk samples such as those used in XRD. Additionally or alternatively, as FEM is more sensitive to higher-order correlations, the lack of graphitic order between planes due to curvature and defects may also have quenched this peak.

The other notable features in the variance pattern for the $\mathrm{C}_{45 \mathrm{O}}$ plot are the sharp spikes at 6.8 $\mathrm{nm}^{-1}$ and $9.5 \mathrm{~nm}^{-1}$. These are due to regions where the structure approaches crystalline perfection within the volume probed. Thus they should not be considered necessarily representative of the overall structure of $\mathrm{C}_{45} \mathrm{O}_{2}$.
Fig. 11(b) shows that the magnitude of the variance increases with activation. The increase in the variance at $4 \mathrm{~nm}^{-1}$ and $8 \mathrm{~nm}^{-1}$ is indicative of increased in-plane ordering over the medium range in $\mathrm{C}_{45} \mathrm{O}_{2}$ compared to $\mathrm{C}$, confirming the findings from the RDF and EELS-SI analysis. This increased ordering may take the form of an increased number density, size or internal order of the regions with correlated structure.
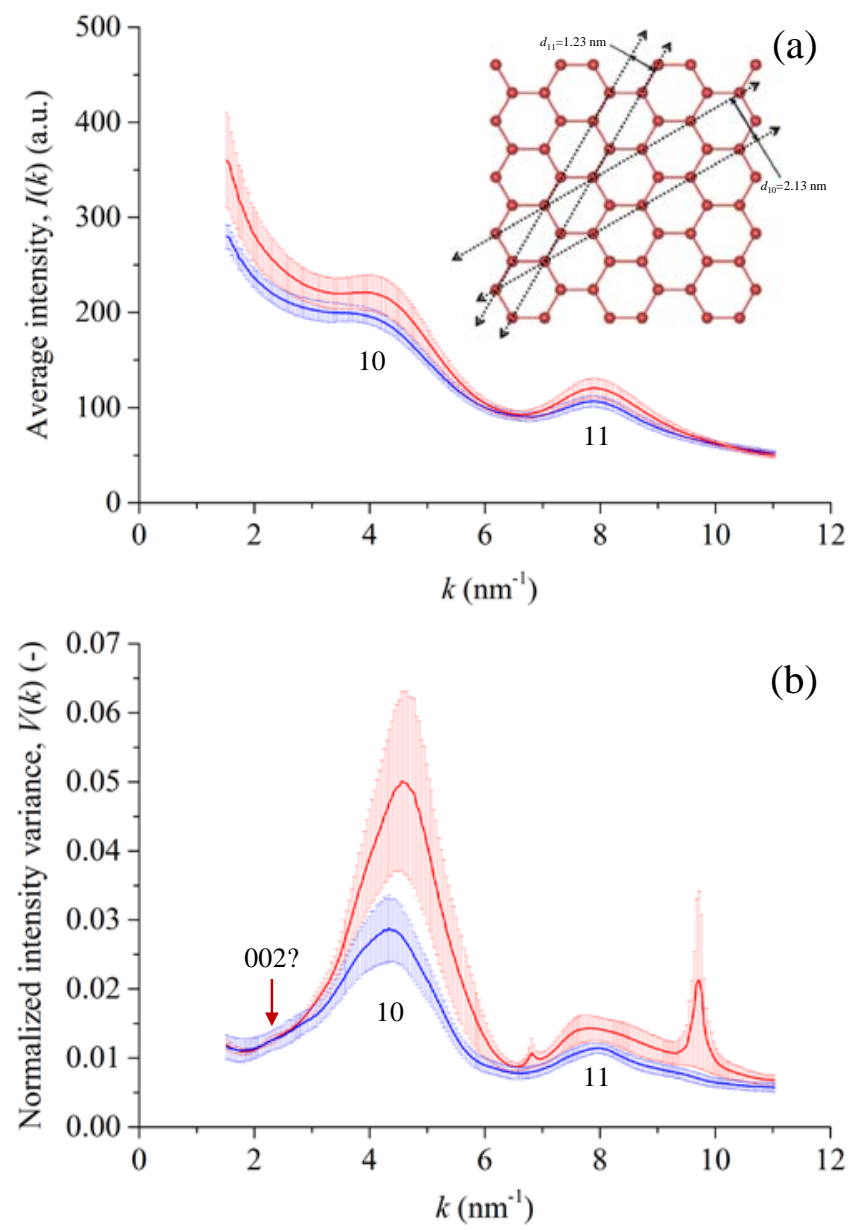

Fig. 11. FEM results for the $\mathrm{C} 0$ (blue) and $\mathrm{C}_{4} \mathrm{O}_{2}$ (red) carbons: (a) azimuthally-averaged electron nanodiffraction pattern; and (b) azimuthallyaveraged intensity variance, Eq. 9. The patterns are based on 3600 separate nanodiffraction patterns each obtained for a $0.5 \times 0.5 \mathrm{~nm}^{2}$ area within the total scanned area (see Fig. 3). 


\subsection{Discussion on evolution of carbon skeleton during activation}

The homogeneity of the TEM images of the unactivated carbon is not inconsistent with its low porosity, a view supported by the excessive time ( $\sim 1$ week) needed to achieve equilibrium in $\mathrm{N}_{2}$ adsorption at $77 \mathrm{~K}$. The TEM image of the $\mathrm{C} 0$ carbon is also suggestive of a solid skeleton that is highly disordered at the short and mesoscales, in line with the EELS-SI analysis, which revealed that substantial fraction (up to $30 \%$ ) of the material is non-sp $p^{2}$ in character with little correlation beyond a nanometre. Taken altogether, these characterizations of the unactivated material are in keeping with the model proposed by Mariwala and Foley [10] based on a range of methods not used here.

Analysis of the XRD patterns of the carbons along the activation pathway using the graphitic crystallite model [2] suggests little change in the skeleton during activation. The remaining analyses undertaken are contrary to this finding, however, suggesting the graphitic crystallite model is inappropriate for PFA-based carbons at the very least.

The helium pycnometry analysis strongly indicates that the density of the solid skeleton of the carbon material studied here densifies along the activation pathway, with it increasing from $\sim 78 \%$ of graphite in the activated material to $~ 93 \%$ after $45 \%$ conversion. The RDF, EELS-SI and FEM analyses all suggest that this densification comes through increased extent of graphenic layers with little increase in graphitic ordering: the first two indicate an increase in the $s p^{2}$ content along the activation pathway, whilst all of these analyses indicate parallel growth in medium range order within the graphene planes.

Taken together, the analyses undertaken in the study reported here strongly point to an oxidative densification of the carbonaceous skeleton of our carbon along the activation pathway. It also suggests that this densification predominately comes through intra-planar re-arrangement to form $s p^{2}$ networks of greater extent - what one might term 'graphenization' rather than graphitization. It is not clear if this occurs via the mechanisms hypothesized many years ago by the pioneers in the field [18, 19, 23] - namely removal of more defective carbon that breaks the restraining cross-links and gives space for subsequent re-arrangement of the carbon in an annealing-type process - but the TEM images do suggest the solid skeleton of the unactivated material is very tightly packed whilst that of the activated material is more open.

\section{Conclusions}

The change in the solid skeleton along the pathway of a carbon activation process was studied using helium pycnometry, synchrotronbased XRD, TEM, EELS-SI, and FEM. Results of this unique combination of methods strongly indicates, when taken together, that the carbon skeleton densifies by in-plane growth of $s p^{2}$ carbon, which might be termed 'graphenization', rather than growth normal to the planes (i.e. graphitization does not occur). Whilst the exact mechanism by which this process occurs cannot be discerned, the TEM images provide some 
support for the long-held belief that graphenization occurs through preferential removal of the more reactive solid atoms to eliminate some cross-linking and create space so as the remaining carbon atoms can move in an annealing process.

Analysis of the XRD patterns of the carbons along the activation pathway using the graphitic crystallite model [2] suggested little change in the carbonaceous skeleton during activation. This is contrary to the results obtained from all the other methods used here, including the RDFs obtained from the same XRD scattering patterns. In line with much criticism of this model over the years [39], it appears as if the graphitic crystallite model is inappropriate for PFA-based carbons studied here.

This report demonstrates some of the potential of EELS-SI, and FEM in the study of nanoporous carbons. In the future we will look to extend the work here to obtain greater quantitative understanding of the growth of in-plane order. We will also look to use the variance derived in FEM - which captures four-body correlation effects - in Virtual Porous Carbon models.

\section{Acknowledgements}

$\mathrm{CH}$ acknowledges a joint scholarship provided by China Scholarship Council (CSC) and the University of Adelaide. The support of the Australian Research Council Discovery Program (DP110101293) is also gratefully acknowledged. ACYL gratefully acknowledges the support of the Science Faculty and the Monash Centre for Electron Microscopy (MCEM), Monash
University. The electron microscopy was performed in the MCEM. The FEI Titan ${ }^{3}$ 80-300 FEGTEM was funded by the Australian Research Council (LE0454166). SHM acknowledges the award of a President's Scholarship from the University of South Australia. The authors thank Tim Petersen for many illuminating discussions. The XRD experiments were undertaken on the powder diffraction beam line at the Australian Synchrotron, Victoria, Australia.

\section{References}

[1] Bandosz T, Biggs M, Gubbins K, Hattori Y, Iiyama T, Kaneko K, et al. Molecular models of porous carbons. In: Radovic L, editor. Chemistry and Physics of Carbon, Vol 28, New York: Marcel Dekker; 2003 p. 41-228.

[2] Biscoe J, Warren BE. An X-ray study of carbon black. J Appl Phys 1942; 13(6):364-71.

[3] Franklin RE. Crystallite growth in graphitizing and non-graphitizing carbons. Proc Roy Soc A 1951; 209(1097):196-218.

[4] Noda T, Inagaki M. Heat treatment of carbon under various pressures. Nature 1962; 196(4856):772-.

[5] Marsh H, Griffiths JA. High resolution electron microscopy study of graphitization of graphitizable carbons. Extended abstracts, International Symposium on Carbon; Toyohashi (Tokyo, Japan); 1982. p. 81-3.

[6] Oberlin A. Carbonization and graphitization. Carbon 1984; 22(6):521-41.

[7] Marsh H, Crawford D. Structure in graphitizable carbon from coal-tar pitch HTT 750-1148 K. Studied using high resolution electron microscopy. Carbon 1984; 22(4-5):41322.

[8] Masters KJ, McEnaney B. The development of structure and microporosity in cellulose carbon. Carbon 1984; 22(6):595-601.

[9] Gruber T, Zerda TW, Gerspacher M. Raman studies of heat-treated carbon blacks. Carbon 1994; 32(7):1377-82. 
[10] Mariwala RK, Foley HC. Evolution of ultramicroporous adsorptive structure in poly(furfuryl alcohol)-derived carbogenic molecular sieves. Ind Eng Chem Res 1994; 33(3):607-15.

[11] Emmerich FG. Evolution with heat treatment of crystallinity in carbons. Carbon 1995; 33(12):1709-15.

[12] Pastor-Villegas J, Durán-Valle CJ, Valenzuela-Calahorro C, Gómez-Serrano V. Organic chemical structure and structural shrinkage of chars prepared from rockrose. Carbon 1998; 36(9):1251-6.

[13] Petkov V, Difrancesco RG, Billinge SJL, Acharya M, Foley HC. Local structure of nanoporous carbons. Philos Mag B 1999; 79(10):1519-30.

[14] Russell NV, Gibbins JR, Williamson J. Structural ordering in high temperature coal chars and the effect on reactivity. Fuel 1999; 78(7):8037.

[15] Feng B, Bhatia SK, Barry JC. Structural ordering of coal char during heat treatment and its impact on reactivity. Carbon 2002; 40(4):481-96.

[16] Burket CL, Rajagopalan R, Marencic AP, Dronvajjala K, Foley HC. Genesis of porosity in polyfurfuryl alcohol derived nanoporous carbon. Carbon 2006; 44(14):2957-63.

[17] Burket CL, Rajagopalan R, Foley HC. Overcoming the barrier to graphitization in a polymer-derived nanoporous carbon. Carbon 2008; 46(3):501-10.

[18] Johnson JL. Relationship between the gasification reactivities of coal char and the physical and chemical properties of coal and coal char. Prepr Am Chem Soc Div Fuel Chem 1975; 20(4):85-101.

[19] Hurt RH, Dudek DR, Longwell JP, Sarofim AF. The phenomenon of gasification-induced carbon densification and its influence on pore structure evolution. Carbon 1988; 26(4):433-49.

[20] Rouzaud JN, Duval B, Leroy J. Coke microtexture: One key for coke reactivity. In: Lahaye J, Ehrburger P, editors. Fundamental Issues in Control of Carbon Gasification Reactivity, Dordrecht, The Netherlands: Kluwer Academic Publishers; 1991 p. 257-67.
[21] Kantorovich II, Bar-Ziv E. Processes in highly porous chars under kinetically controlled conditions: I. Evolution of the porous structure. Combust Flame 1994; 97(1):61-78.

[22] Feng B, Bhatia SK, Barry JC. Variation of the crystalline structure of coal char during gasification. Energy Fuels 2003; 17(3):744-54.

[23] Noda T, Inagaki M. Effect of gas phase on graphitization of carbon. Carbon 1964; 2(2):12730.

[24] Levendis YA, Flagan RC. Synthesis, formation and characterization of micron-sized glassy carbon spheres of controlled pore structure. Carbon 1989; 27(2):265-83.

[25] Johnson JL. Fundamentals of coal gasification. In: Elliott MA, editor. Chemistry of Coal Utilization: Second supplementary volume, New York: Wiley; 1981 p. 1491-598.

[26] Kantorovich II, Bar-Ziv E. Processes in highly porous chars under kinetically controlled conditions: II. Pore reactivity. Combust Flame 1994; 97(1):79-87.

[27] Kantorovich II, Bar-ziv E. The effect of microstructural transformation on the evolution of thermal conductivity of highly porous chars during oxidation. Combust Flame 1997; 109(4):521-35.

[28] Furuta T, Yamashita Y, Shiraishi M. Microtextural change in carbons by reaction with $\mathrm{CO}_{2}$ gas. Tanso 1989; 140:241-7.

[29] Sha X-Z, Kyotani T, Tomita A. Rate retardation phenomenon during gasification of Wandoan coal char. Fuel 1990; 69(12):1564-7.

[30] Davis KA, Hurt RH, Yang NYC, Headley TJ. Evolution of char chemistry, crystallinity, and ultrafine structure during pulverized-coal combustion. Combust Flame 1995; 100(1-2):3140.

[31] Hurt RH, Davis KA, Yang NYC, Headley TJ, Mitchell GD. Residual carbon from pulverizedcoal-fired boilers. 2. Morphology and physicochemical properties. Fuel 1995; 74(9):1297-306.

[32] Palotás ÁB, Rainey LC, Sarofim AF, Vander Sande JB, Ciambelli P. Effect of oxidation on the microstructure of carbon blacks. Energy Fuels 1996; 10(1):254-9. 
[33] Sharma A, Kyotani T, Tomita A. A new quantitative approach for microstructural analysis of coal char using HRTEM images. Fuel 1999; 78(10):1203-12.

[34] Shim H-S, Hurt RH, Yang NYC. A methodology for analysis of 002 lattice fringe images and its application to combustion-derived carbons. Carbon 2000; 38(1):29-45.

[35] Sharma A, Kadooka H, Kyotani T, Tomita A. Effect of microstructural changes on gasification reactivity of coal chars during low temperature gasification. Energy Fuels 2001; 16(1):54-61.

[36] Lu LM, Kong CH, Sahajwalla V, Harris D. Char structural ordering during pyrolysis and combustion and its influence on char reactivity. Fuel 2002; 81(9):1215-25.

[37] Oberlin A, Oberlin M. Graphitizability of carbonaceous materials as studied by TEM and X-ray diffraction. J Microsc 1983; 132(3):353-63.

[38] Tran KN, Berkovich AJ, Tomsett A, Bhatia SK. Crystalline structure transformation of carbon anodes during gasification. Energy Fuels 2008; 22(3):1902-10.

[39] Marsh H, Rodríguez-Reinoso F. Activated Carbon. Oxford: Elsevier Science Ltd; 2006.

[40] Harris PJF, Liu Z, Suenaga K. Imaging the atomic structure of activated carbon. J Phys: Condens Matter 2008; 20(36).

[41] Guo J, Morris JR, Ihm Y, Contescu CI, Gallego NC, Duscher G, et al. Topological defects: Origin of nanopores and enhanced adsorption performance in nanoporous carbon. Small 2012; 8(21):3283-8.

[42] Shi H, Reimers JN, Dahn JR. Structurerefinement program for disordered carbons. J Appl Cryst 1993; 26(6):827-36.

[43] Egami T, Billinge SJL. Underneath the Bragg Peaks: Structural Analysis of Complex Materials. 2nd ed. New York: Pergamon; 2012.

[44] Fan GY, Cowley JM. The simulation of high resolution images of amorphous thin films. Ultramicroscopy 1987; 21(2):125-30.

[45] Treacy MMJ, Gibson JM, Fan L, Paterson DJ, McNulty I. Fluctuation microscopy: a probe of medium range order. Rep Prog Phys 2005; 68(12):2899.
[46] Liu ACY, Arenal R, Miller DJ, Chen X, Johnson JA, Eryilmaz OL, et al. Structural order in near-frictionless hydrogenated diamondlike carbon films probed at three length scales via transmission electron microscopy. Phys Rev B 2007; 75(20):205402.

[47] Chen X, Sullivan JP, Friedmann TA, Gibson JM. Fluctuation microscopy studies of mediumrange ordering in amorphous diamond-like carbon films. Appl Phys Lett 2004; 84(15):28235.

[48] Zhao G, Buseck PR, Rougée A, Treacy MMJ. Medium-range order in molecular materials: Fluctuation electron microscopy for detecting fullerenes in disordered carbons. Ultramicroscopy 2009; 109(2):177-88.

[49] Ishiguro T, Suzuki N, Fujitani Y, Morimoto H. Microstructural changes of diesel soot during oxidation. Combust Flame 1991; 85(1-2):1-6.

[50] Jeanne-Rose V, Golabkan V, Mansot JL, Largitte L, Césaire T, Ouensanga A. An EELSbased study of the effects of pyrolysis on natural carbonaceous materials used for activated charcoal preparation. J Microsc 2003; 210(1):539.

[51] Laffont L, Monthioux M, Serin V, Mathur RB, Guimon C, Guimon MF. An EELS study of the structural and chemical transformation of PAN polymer to solid carbon. Carbon 2004; 42(12-13):2485-94.

[52] Daniels H, Brydson R, Rand B, Brown A. Investigating carbonization and graphitization using electron energy loss spectroscopy (EELS) in the transmission electron microscope (TEM). Philos Mag 2007; 87(27):4073-92.

[53] Zhang Z-l, Brydson R, Aslam Z, Reddy S, Brown A, Westwood A, et al. Investigating the structure of non-graphitising carbons using electron energy loss spectroscopy in the transmission electron microscope. Carbon 2011; 49(15):5049-63.

[54] Hu C, Sedghi S, Madani SH, SilvestreAlbero A, Sakamoto H, Kwong P, et al. Control of the pore size distribution and its spatial homogeneity in particulate activated carbon. Carbon 2014; 78(0):113-20.

[55] This nomenclature is identical to that used in [52] and related reports to follow. 
[56] Sedghi S, Madani SH, Hu C, SilvestreAlberoc A, Kwong P, Pendleton P, et al. Control of the spatial homogeneity of pore surface chemistry in particulate activated carbon. In preparation.

[57] Neimark AV, Lin Y, Ravikovitch PI, Thommes M. Quenched solid density functional theory and pore size analysis of micromesoporous carbons. Carbon 2009; 47(7):161728.

[58] Rouquerol J, Llewellyn P, Rouquerol F. Is the BET equation applicable to microporous adsorbents? In: P.L. Llewellyn FR-RJR, Seaton N, editors. Studies in Surface Science and Catalysis, Volume 160: Elsevier; 2007 p. 49-56.

[59] Qajar A, Peer M, Rajagopalan R, Liu Y, Brown C, Foley HC. Surface compression of light adsorbates inside microporous PFA-derived carbons. Carbon 2013; 60(0):538-49.

[60] Bergamaschi A, Cervellino A, Dinapoli R, Gozzo F, Henrich B, Johnson I, et al. Photon counting microstrip detector for time resolved powder diffraction experiments. Nucl Instrum Meth A 2009; 604(1-2):136-9.

[61] Haverkamp RG, Wallwork KS. X-ray pair distribution function analysis of nanostructured materials using a Mythen detector. J Synchrotron Radiat 2009; 16(6):849-56.

[62] Qiu X, Thompson JW, Billinge SJL. PDFgetX2: a GUI-driven program to obtain the pair distribution function from X-ray powder diffraction data. J Appl Cryst 2004; 37(4):678-.

[63] Gregg SJ, Sing KSW. Adsorption, Surface Area and Porosity. 2nd ed. London: Academic Press; 1982.

[64] Pikunic J, Clinard C, Cohaut N, Gubbins KE, Guet J-M, Pellenq RJM, et al. Structural modeling of porous carbons: Constrained reverse monte carlo method. Langmuir 2003; 19(20):8565-82.

[65] Dmowski W, Contescu CI, Llobet A, Gallego NC, Egami T. Local atomic density of microporous carbons. J Phys Chem C 2011; 116(4):2946-51.

[66] Ruike M, Kasu T, Setoyama N, Suzuki T, Kaneko K. Inaccessible pore characterization of less-crystalline microporous solids. J Phys Chem 1994; 98(38):9594-600.
[67] Scherdel C, Reichenauer G. The impact of residual adsorbate on the characterization of microporous carbons with small angle scattering. Carbon 2012; 50(8):3074-82.

[68] Berger SD, McKenzie DR, Martin PJ. EELS analysis of vacuum arc-deposited diamond-like films. Philos Mag Lett 1988; 57(6):285-90.

[69] Suenaga K, Koshino M. Atom-by-atom spectroscopy at graphene edge. Nature 2010; 468(7327):1088-90.

[70] Zobelli A, Gloter A, Ewels CP, Seifert G, Colliex C. Electron knock-on cross section of carbon and boron nitride nanotubes. Phys Rev B 2007; 75(24):245402.

[71] Hébert C, Schattschneider P, Franco H, Jouffrey B. ELNES at magic angle conditions. Ultramicroscopy 2006; 106(11-12):1139-43.

[72] Egerton RF. Electron Energy-Loss Spectroscopy in the Electron Microscope. Springer US; 2011.

[73] Voyles PM, Muller DA. Fluctuation microscopy in the STEM. Ultramicroscopy 2002; 93(2):147-59.

[74] Dahn JR, Xing W, Gao Y. The "falling cards model” for the structure of microporous carbons. Carbon 1997; 35(6):825-30.

[75] Smith MA, Foley HC, Lobo RF. A simple model describes the PDF of a non-graphitizing carbon. Carbon 2004; 42(10):2041-8.

[76] Farmahini AH, Opletal G, Bhatia SK. Structural modelling of silicon carbide-derived nanoporous carbon by hybrid reverse monte carlo simulation. J Phys Chem C 2013; 117(27):1408194.

[77] Hawelek L, Brodka A, Dore JC, Honkimäki V, Burian A. Fullerene-like structure of activated carbons. Diamond Relat Mater 2008; 17(710):1633-8.

[78] Yazyev OV, Louie SG. Topological defects in graphene: Dislocations and grain boundaries. Phys Rev B 2010; 81(19).

[79] Banhart F, Kotakoski J, Krasheninnikov AV. Structural defects in graphene. ACS Nano 2010; 5(1):26-41.

[80] O’Malley B, Snook I, McCulloch D. Reverse Monte Carlo analysis of the structure of glassy 
carbon using electron-microscopy data. Phys Rev B 1998; 57(22):14148-57.

[81] Pikunic J, Pellenq RJM, Thomson KT, Rouzaud JN, Levitz P, Gubbins KE. Improved molecular models for porous carbons. In: Iwasawa $\mathrm{Y}$, Oyama $\mathrm{N}$, Kunieda $\mathrm{H}$, editors. Studies in Surface Science and Catalysis, Volume 132: Elsevier; 2001 p. 647-52.

[82] Per Z, Sigita U, Fredrik L, Robert GD, Jaan L, Gunnar S. Reverse Monte Carlo studies of nanoporous carbon from TiC. J Phys: Condens Matter 2005; 17(23):3509.

[83] Burian A, Ratuszna A, Dore JC. Radial distribution function analysis of the structure of activated carbons. Carbon 1998; 36(11):1613-21.

[84] Temleitner L, Pusztai L, Rubio-Arroyo MF, Aguilar-López S, Klimova T, Pizio O. Microscopic and mesoscopic structural features of an activated carbon sample, prepared from sorghum via activation by phosphoric acid. Mater Res Bull 2012; 47(12):4409-13.

[85] Castro-Marcano F, Winans RE, Chupas P, Chapman K, Calo JM, Watson JK, et al. Fine structure evaluation of the pair distribution function with molecular models of the Argonne premium coals. Energy Fuels 2012; 26(7):433645.

[86] Urbonaite S, Wachtmeister S, Mirguet C, Coronel E, Zou WY, Csillag S, et al. EELS studies of carbide derived carbons. Carbon 2007; 45(10):2047-53.

[87] The t-test and F-test were used to test for similarity or otherwise of the means and variances respectively of the distributions of $s p^{2}$ fraction.

[88] Song CW, Wang TH, Wang XY, Qiu JS, Cao YM. Preparation and gas separation properties of poly(furfuryl alcohol)-based C/CMS composite membranes. Sep Purif Technol 2008; 58(3):412-8. 